\title{
Operators with Singular Trace Conditions on a Manifold with Edges
}

\author{
D. Kapanadze, B.-W. Schulze, and J. Seiler
}

\begin{abstract}
We establish a new calculus of pseudodifferential operators on a manifold with smooth edges and study ellipticity with extra trace and potential conditions (as well as Green operators) at the edge. In contrast to the known scenario with conditions of that kind in integral form we admit in this paper 'singular' trace, potential and Green operators, which are related to the corresponding operators of positive type in Boutet de Monvel's calculus for boundary value problems.
\end{abstract}

\section{VERSION: January 18, 2006}

\section{Contents}

1. Introduction 1

2. Symbols of Boutet de Monvel's algebra 3

3. Symbolic structure of edge-degenerate differential operators 5

4. Spaces with asymptotics 8

5. The class of edge symbols of non-trivial type 11

6. Operators of non-trivial type on manifolds with edges 20

7. Ellipticity and parametrix 25

8. Appendix: The standard calculus for manifolds with edges 29

References

\section{Introduction}

The construction of a pseudodifferential algebra containing both the classical boundary value problems (such as the Dirichlet or Neumann problem for the Laplacian) as well as their parametrices leads in case of a (compact) manifold $M$ with smooth boundary to Boutet de Monvel's algebra [1].

2000 Mathematics Subject Classification. 35J40, 35J70, 47G30, 58J40.

Key words and phrases. Operators on manifolds with edges, singular trace and Green operators, ellipticity and parametrices of edge problems. 
The operators in this calculus have block-matrix form,

$$
\left(\begin{array}{cc}
A & K \\
T & Q
\end{array}\right): \begin{array}{ccc}
\mathcal{C}^{\infty}(M, E) & \oplus \\
\mathcal{C}^{\infty}\left(\partial M, J_{-}\right) & \begin{array}{c}
\mathcal{C}^{\infty}(M, F) \\
\mathcal{C}^{\infty}\left(\partial M, J_{+}\right)
\end{array}
\end{array},
$$

(which continuously extend to Sobolev spaces), where $E, F$ and $J_{-}, J_{+}$are vector bundles over $M$ and the boundary $\partial M$, respectively. Classical boundary value problems correspond to operators $\left(\begin{array}{l}A \\ T\end{array}\right)$ and $\operatorname{dim} J_{-}=0$, where $A$ is a differential operator and $T$ the boundary condition, now called a trace operator. Shapiro-Lopatinskij elliptic problems have a parametrix of the form $(A K)$, where $K$ is a potential operator and $A=\mathrm{r}^{+} P \mathrm{e}^{+}+G$ with a (singular) Green operator $G$ and a classical pseudodifferential operator $P$ on the double $2 M$ of $M$ that has the transmission property with respect to the boundary of $M$ (moreover, $\mathrm{e}^{+}$denotes the operator of extension by zero from $M$ to $2 M$, while $\mathrm{r}^{+}$denotes restriction from $2 M$ to int $M$ ). Elements of Boutet de Monvel's algebra are filtered by their order $\mu \in \mathbb{Z}$ (which is simply the usual order of the (pseudo)differential part in the upper left corner) and posses a so-called type, which is a non-negative integer $d \in \mathbb{N}_{0}$. The type can be different from zero only for trace operators and (singular) Green operators. For example, taking $k$-times the derivative in normal direction followed by restriction to the boundary and application of a pseudodifferential operator on the boundary yields a trace operator of type $d=k+1$.

Another more complicated task is that of developing an elliptic theory for differential operators on a manifold $M$ with edges. Roughly speaking, outside a lower dimensional smooth stratum $Y$ (the edge), $M$ is a smooth manifold, while each point of $Y$ has a neighbourhood homeomorphic to $U \times X^{\Delta}$, where $U$ is an open subset of $Y$ and $X^{\Delta}$ is a cone with smooth closed cross section $X$. Note that a manifold with boundary is a particular case of a manifold with edge, namely one with $X$ being a point and thus $X^{\Delta}=\overline{\mathbb{R}}_{+}$, the normal to the boundary. Typical differential operators on $M$ are edge degenerate, i.e. they have a specific singular structure close to the edge (see Section 3 for more details). As it turns out, elliptic edge degenerate differential operators in general do not have the Fredholm property (in suitable edge Sobolev spaces), but one has to impose additional trace and potential conditions over the edge, possibly even simultaneously. In other words, developing a Fredholm theory for edge degenerate operators leads to a pseudodifferential calculus of operators in block-matrix form similarly as in (1.1), the so-called edge algebra. This calculus has been developed by the first author in [10], [11], [12]. A detailed exposition may be found in $[\mathbf{3}]$; a short overview we provide in Section 8 of this paper.

As is known from the author's joint works $[\mathbf{1 3}]$ or $[\mathbf{1 4}]$ the analogy between the respective calculi for boundary value problems and 'edge problems' goes very far. But there are also essential differences: While Boutet de Monvel's calculus requires the transmission property, the edge calculus admits general pseudodifferential symbols smooth up to the boundary. In contrast to standard elliptic trace conditions (e.g. of Dirichlet or Neumann type) at the boundary which make sense on solutions of sufficiently high Sobolev regularity, the solutions to edge problems generally do not admit traces of that kind. On the other hand, solutions to edge problems may have asymptotics at the edge, that can be regarded as a substitute of Taylor asymptotics at the boundary (i.e., smoothness up to the boundary). It is now natural and desirable to organise a calculus of edge degenerate operators with trace conditions based on those general asymptotics (called singular trace conditions) rather than Taylor asymptotics. 
In the present paper we just establish such an extension of the edge algebra by our new singular trace and Green operators. We study compositions of the corresponding edge operators, formulate ellipticity with singular trace, etc., conditions at the edge, and obtain parametrices within the calculus.

\section{Symbols of Boutet de Monvel's algebra}

As noted in the introduction, boundary value problems with the transmission property may be regarded as a model for calculi with singular trace (and also Green) operators. Let us describe this on the level of boundary symbols in a half-space $\Omega \times \overline{\mathbb{R}}_{+}$with an open set $\Omega \subset \mathbb{R}^{q}$. The variable in $\Omega$ we will denote by $y$, the one of $\overline{\mathbb{R}}_{+}$by $t$.

Let $p(y, t, \eta, \tau)$ be a (classical) symbol of integer order $\mu$ with the transmission property at $t=0$ (the symbols of that kind form a closed subspace $S_{\mathrm{tr}}^{\mu}\left(\Omega \times \overline{\mathbb{R}}_{+} \times \mathbb{R}^{q+1}\right)$ of $C^{\infty}\left(\Omega \times \overline{\mathbb{R}}_{+}, S_{\mathrm{cl}}^{\mu}\left(\mathbb{R}^{q+1}\right)\right)$ with $S_{\mathrm{cl}}^{\mu}\left(\mathbb{R}^{q+1}\right)$ being the space of classical symbols of order $\mu$ with constant coefficients). Assume that $p$ is independent of $t$ for $t>T$ for some $T>0$. Let us set

$$
\mathrm{op}^{+}(p)(y, \eta)=\mathrm{r}^{+} \mathrm{op}(p)(y, \eta) \mathrm{e}^{+}
$$

for $(\operatorname{op}(p)(y, \eta) u)(t)=\iint e^{i\left(t-t^{\prime}\right) \tau} p(y, t, \eta, \tau) u\left(t^{\prime}\right) d t^{\prime} d \tau, d \tau=(2 \pi)^{-1} d \tau$, where $\mathrm{e}^{+}$denotes the operator of extension by zero from $\mathbb{R}_{+}$to $\mathbb{R}$ and $\mathrm{r}^{+}$the restriction from $\mathbb{R}$ to $\mathbb{R}_{+}$of distributions (in (2.1) on the right hand side we tacitly insert a symbol $\widetilde{p}$ on $\Omega \times \mathbb{R} \times \mathbb{R}^{q+1}$ with $p=\left.\widetilde{p}\right|_{\Omega \times \overline{\mathbb{R}}_{+} \times \mathbb{R}^{q+1}}$; however, since the operator is independent of the choice of $\tilde{p}$ we simply write $p)$. As is known, (2.1) defines a family of continuous operators

$$
\mathrm{op}^{+}(p)(y, \eta): H^{s}\left(\mathbb{R}_{+}\right) \longrightarrow H^{s-\mu}\left(\mathbb{R}_{+}\right), \quad s>-\frac{1}{2},
$$

where $H^{s}\left(\mathbb{R}_{+}\right):=\left.H^{s}(\mathbb{R})\right|_{\mathbb{R}_{+}}$with $H^{s}(\mathbb{R})$ being the standard Sobolev space of smoothness $s$ on $\mathbb{R}$. Defining for $\lambda>0$

$$
\kappa_{\lambda}: H^{s}\left(\mathbb{R}_{+}\right) \longrightarrow H^{s}\left(\mathbb{R}_{+}\right), \quad\left(\kappa_{\lambda} u\right)(t)=\lambda^{\frac{1}{2}} u(\lambda t),
$$

we obtain a strongly continuous group of isomorphisms on the space $H^{s}\left(\mathbb{R}_{+}\right)$, i.e.,

i) $\kappa_{1}=1$ and $\kappa_{\lambda} \kappa_{\delta}=\kappa_{\lambda \delta}$ for all $\lambda, \delta>0$,

ii) $\lambda \mapsto \kappa_{\lambda} u:[0, \infty) \rightarrow H^{s}\left(\mathbb{R}_{+}\right)$is continuous for any $u \in H^{s}\left(\mathbb{R}_{+}\right)$.

In particular, these operators are unitary on $L^{2}\left(\mathbb{R}_{+}\right)$(with the standard scalar product). An essential observation is that $\mathrm{op}^{+}(p)(y, \eta)$ is an operator-valued symbol in the following sense.

If $E$ is a Hilbert space and $\left\{\kappa_{\lambda}\right\}_{\lambda \in \mathbb{R}_{+}}$a strongly continuous group of isomorphisms on $E$, we say that $E$ is endowed with a group action. Let us set $\langle\eta\rangle=\left(1+|\eta|^{2}\right)^{1 / 2}$.

Definition 2.1. Given Hilbert spaces $E$ and $\widetilde{E}$ with group actions $\left\{\kappa_{\lambda}\right\}_{\lambda \in \mathbb{R}_{+}}$and $\left\{\tilde{\kappa}_{\lambda}\right\}_{\lambda \in \mathbb{R}_{+}}$, respectively,

$$
S^{\mu}\left(\Omega \times \mathbb{R}^{q} ; E, \widetilde{E}\right)
$$

will denote the space of all functions $a(y, \eta) \in C^{\infty}\left(\Omega \times \mathbb{R}^{q}, \mathcal{L}(E, \widetilde{E})\right)$ such that

$$
\sup _{y \in K, \eta \in \mathbb{R}^{q}}\langle\eta\rangle^{-\mu+|\alpha|}\left\|\tilde{\kappa}_{\langle\eta\rangle}^{-1} D_{\eta}^{\alpha} D_{y}^{\beta} a(y, \eta) \kappa_{\langle\eta\rangle}\right\|_{\mathcal{L}(E, \widetilde{E})}<\infty
$$

for every compact $K \subset \Omega$ and all multi-indices $\alpha, \beta \in \mathbb{N}^{q}$. 
Note that we obtain an equivalent definition of (2.3) when we replace $\langle\eta\rangle$ by, for instance, a smooth function $\eta \mapsto[\eta]$ that is strictly positive and satisfies $[\eta]=|\eta|$ for $|\eta|>C$ for a $C>0$. In the sequel, for convenience, we assume $C=1$.

A function $a_{(\mu)}(y, \eta) \in C^{\infty}\left(\Omega \times\left(\mathbb{R}^{q} \backslash\{0\}\right), \mathcal{L}(E, \widetilde{E})\right)$ is called (twisted) homogeneous in $\eta \neq 0$ of order $\mu$ if

$$
a_{(\mu)}(y, \lambda \eta)=\lambda^{\mu} \tilde{\kappa}_{\lambda} a_{(\mu)}(y, \eta) \kappa_{\lambda}^{-1} \quad \text { for all }(y, \eta) \in \Omega \times\left(\mathbb{R}^{q} \backslash\{0\}\right) \quad \text { for all } \lambda>0 .
$$

Note that when $\chi(\eta)$ is an arbitrary zero-excision function on $\mathbb{R}^{q}$ (i.e., $\chi$ is smooth, vanishes in a neighbourhood of the origin, and equals 1 for $|\eta|>R$ for some $R>0)$ we have $\chi(\eta) a_{(\mu)}(y, \eta) \in$ $S^{\mu}\left(\Omega \times \mathbb{R}^{q} ; E, \widetilde{E}\right)$ if $a_{(\mu)}$ is homogeneous in the former sense. This gives rise to

$$
S_{\mathrm{cl}}^{\mu}\left(\Omega \times \mathbb{R}^{q} ; E, \widetilde{E}\right),
$$

the subspace of $(2.3)$ of all elements $a(y, \eta)$ which admit an asymptotic expansion into terms of the kind $\chi(\eta) a_{(\mu-j)}(y, \eta)$, with homogeneous functions $a_{(\mu-j)}(y, \eta)$ of order $\mu-j, j \in \mathbb{N}$. In this case we let

$$
\sigma_{\wedge}(a)(y, \eta)=a_{(\mu)}(y, \eta)
$$

denote the homogeneous principal symbol.

The concept of operator-valued symbols in the above sense is very close to the scalar case where $E=\widetilde{E}=\mathbb{C}$ and the group actions are trivial (i.e., identity operators for all $\lambda>0$ ). Nevertheless there are beautiful (and sometimes surprising) examples of such operator-valued symbols, as we shall see below.

EXAmPLE 2.2. For every $p(y, t, \eta, \tau) \in S_{\mathrm{tr}}^{\mu}\left(\Omega \times \overline{\mathbb{R}}_{+} \times \mathbb{R}^{q+1}\right)$ (independent of $t$ for large $t$ ) we have

$$
\mathrm{op}^{+}(p)(y, \eta) \in S^{\mu}\left(\Omega \times \mathbb{R}^{q} ; H^{s}\left(\mathbb{R}_{+}\right), H^{s-\mu}\left(\mathbb{R}_{+}\right)\right), \quad s>-\frac{1}{2} .
$$

Moreover, if $p_{(\mu)}$ denotes the homogeneous principal part of $p$ of order $\mu$ in $(\eta, \tau) \neq 0$, then

$$
p_{(\mu)}(y, \eta):=\operatorname{op}^{+}\left(\left.p_{(\mu)}\right|_{t=0}\right)(y, \eta), \quad(y, \eta) \in \Omega \times\left(\mathbb{R}^{q} \backslash\{0\}\right)
$$

is homogeneous in the sense of the relation (2.4).

It is also necessary for our purposes to admit $\widetilde{E}$ to be a Fréchet space with group action: Let

$$
\widetilde{E}=\lim _{j \in \mathbb{N}} \widetilde{E}^{j}
$$

be a projective limit of Hilbert spaces $\widetilde{E}^{j}$ with continuous embeddings $\widetilde{E}^{j+1} \hookrightarrow \widetilde{E}^{j} \hookrightarrow \ldots \hookrightarrow \widetilde{E}^{0}$ such that $\widetilde{E}^{0}$ is endowed with a group action $\left\{\kappa_{\lambda}\right\}_{\lambda \in \mathbb{R}_{+}}$that restricts to a group action $\left\{\left.\kappa_{\lambda}\right|_{\widetilde{E}^{j}}\right\}_{\lambda \in \mathbb{R}_{+}}$ on $\widetilde{E}^{j}$ for every $j$. Then $\widetilde{E}$ is said to be endowed with the group action $\left\{\kappa_{\lambda}\right\}_{\lambda \in \mathbb{R}_{+}}$. We have the spaces $S_{(\mathrm{cl})}^{\mu}\left(\Omega \times \mathbb{R}^{q} ; E, \widetilde{E}^{j}\right)$ for all $j$, and

$$
S_{(\mathrm{cl})}^{\mu}\left(\Omega \times \mathbb{R}^{q} ; E, \widetilde{E}\right)=\lim _{j \in \mathbb{N}} S_{(\mathrm{cl})}^{\mu}\left(\Omega \times \mathbb{R}^{q} ; E, \widetilde{E}^{j}\right)
$$

is, by definition, the projective limit of these spaces (subscript ' $(\mathrm{cl})$ ' means that we are talking about the classical or the general case).

As is known, the parametrix of an elliptic boundary value problem $\left(\begin{array}{l}A \\ T\end{array}\right)$ in the half-space $\Omega \times$ $\mathbb{R}_{+}$, with an elliptic differential operator $A$ and a trace operator $T$ which satisfies the ShapiroLopatinskij condition with respect to $A$ (for instance, the Dirichlet or the Neumann problem for 
the Laplacian), can be expressed within Boutet de Monvel's calculus of pseudodifferential boundary value problems. Besides the symbols of Example 2.2, this calculus also contains so-called Green symbols. Such symbols have an order $\mu \in \mathbb{R}$ and a so-called type $d \in \mathbb{N}$. They have the form of $2 \times 2$ block matrices

$$
g(y, \eta)=g_{0}(y, \eta)+\sum_{l=1}^{d} g_{l}(y, \eta)\left(\begin{array}{cc}
\partial_{r}^{l} & 0 \\
0 & 0
\end{array}\right)
$$

where $\partial_{r}$ indicates differentiation in the direction normal to the boundary, and, with suitable $j_{+}, j_{-} \in \mathbb{N}_{0}$,

$$
g_{l}(y, \eta) \in S_{\mathrm{cl}}^{\nu}\left(\Omega \times \mathbb{R}^{q} ; L^{2}\left(\mathbb{R}_{+}\right) \oplus \mathbb{C}^{j_{-}}, \mathcal{S}\left(\overline{\mathbb{R}}_{+}\right) \oplus \mathbb{C}^{j_{+}}\right), \quad \nu=\mu-l,
$$

is a $2 \times 2$ block matrix symbol of order $\nu$, whose pointwise adjoint satisfies

$$
g_{l}(y, \eta)^{*} \in S_{\mathrm{cl}}^{\nu}\left(\Omega \times \mathbb{R}^{q} ; L^{2}\left(\mathbb{R}_{+}\right) \oplus \mathbb{C}^{j_{+}}, \mathcal{S}\left(\overline{\mathbb{R}}_{+}\right) \oplus \mathbb{C}^{j_{-}}\right) .
$$

Here, $\mathcal{S}\left(\overline{\mathbb{R}}_{+}\right)=\varliminf_{k \in \mathbb{N}}\langle t\rangle^{-k} H^{k}\left(\mathbb{R}_{+}\right)$and $L^{2}\left(\mathbb{R}_{+}\right)$are endowed with the group action $\left\{\kappa_{\lambda}\right\}_{\lambda \in \mathbb{R}_{+}}$ from $(2.2)$, while the spaces $L^{2}\left(\mathbb{R}_{+}\right) \oplus \mathbb{C}^{N}$ and $\mathcal{S}\left(\overline{\mathbb{R}}_{+}\right) \oplus \mathbb{C}^{N}$ are endowed with $\left\{\kappa_{\lambda} \oplus \operatorname{id}_{\mathbb{C}^{N}}\right\}_{\lambda \in \mathbb{R}_{+}}$.

Writing $g(y, \eta)=\left(g_{i j}(y, \eta)\right)_{i, j=1,2}$, the component $g_{21}$ (the left lower corner) is a so-called trace symbol of order $\mu$ and type $d$, while $g_{12}$ is a potential symbol of order $\mu$.

The associated operators $\mathrm{Op}(g)$ (the pseudodifferential operator with respect to the Fourier transform in the $y$-variables), occasionally also denoted by $\mathrm{Op}_{y}(g)$, are generated in the parametrix construction of elliptic boundary value problems. For example, Green's function of the Dirichlet problem for the Laplacian $\Delta$ is, locally near the boundary, of the form $E+\operatorname{Op}\left(g_{11}\right)$ for a fundamental solution $E$ of $\Delta$ and a Green symbol $g_{11}(y, \eta)$ of type 0 . Moreover, the potential operator in the solution of the Dirichlet problem is, locally near the boundary, of the form $\operatorname{Op}\left(g_{12}\right)$ (clearly, these relations are true modulo smoothing operators in Boutet de Monvel's calculus, cf. [1]).

REMARK 2.3. The amplitude functions of boundary value problems in $\Omega \times \mathbb{R}_{+}$have the form

$$
a(y, \eta)=\left(\begin{array}{cc}
\mathrm{op}^{+}(p)(y, \eta) & 0 \\
0 & 0
\end{array}\right)+g(y, \eta)
$$

where $\mathrm{op}^{+}(p)$ is as in Remark 2.2, and the second summand, given by (2.6) belongs to

$$
S_{\mathrm{cl}}^{\mu}\left(\Omega \times \mathbb{R}^{q} ; H^{s}\left(\mathbb{R}_{+}\right) \oplus \mathbb{C}^{j_{-}}, \mathcal{S}\left(\overline{\mathbb{R}}_{+}\right) \oplus \mathbb{C}^{j_{+}}\right), \quad \text { for all } s>d-\frac{1}{2} .
$$

From (2.6) we see that trace and Green operators of type 0 are of integral form (in contrast to operators of positive type that are combined with differentiations in normal direction and the restriction to the boundary). As noted in the introduction, the calculus of edge problems of [12] has a similar structure, but the corresponding analogues of trace and Green operators of positive type are still missing. In the present paper we introduce them as so-called singular operators while the former ones from the edge calculus are of integral form and regarded as regular operators.

\section{Symbolic structure of edge-degenerate differential operators}

We now start discussing a category of operator-valued symbols that contribute to the symbolic calculus of operators on a manifold with edge. By definition (cf. Section 6.1 below) such a manifold is locally, near the edge, represented by a wedge

$$
X^{\triangle} \times \Omega, \quad X^{\triangle}=\left(\overline{\mathbb{R}}_{+} \times X\right) /(\{0\} \times X),
$$


with an edge $\Omega$ (an open set in $\mathbb{R}^{q}$ ) and a model cone $X^{\triangle}$ for a closed smooth Riemannian manifold $X$. The half-space case just corresponds to $\operatorname{dim} X=0$.

An edge-degenerate differential operator $A$ on an open stretched wedge

$$
X^{\wedge} \times \Omega, \quad X^{\wedge}=\mathbb{R}_{+} \times X,
$$

in the splitting of variables $(r, x, y)$ has the form

$$
A=r^{-\mu} \sum_{j+|\alpha| \leq \mu} a_{j \alpha}(r, y)\left(-r \partial_{r}\right)^{j}\left(r D_{y}\right)^{\alpha}
$$

with coefficients $a_{j \alpha} \in C^{\infty}\left(\overline{\mathbb{R}}_{+} \times \Omega\right.$, $\left.\operatorname{Diff}^{\mu-(j+|\alpha|)}(X)\right)$, where $\operatorname{Diff}^{\nu}(X)$ is the space of all differential operators of order $\nu$ with smooth coefficients on $X$. Since the main aspects concern a neighbourhood of $r=0$ we assume that the coefficients $a_{j \alpha}$ are independent of $r$ for $r>R$ for some $R>0$.

We have $A=\mathrm{Op}_{y}(a)$ for the operator-valued amplitude function

$$
a(y, \eta)=r^{-\mu} \sum_{j+|\alpha| \leq \mu} a_{j \alpha}(r, y)\left(-r \partial_{r}\right)^{j}(r \eta)^{\alpha} .
$$

We now fix an adequate scale of spaces such that (3.2) is an operator-valued symbol in the sense of (2.3). To this end we let $\omega$ denote a cut-off function on the half-axis, i.e. $\omega \in C_{0}^{\infty}\left(\overline{\mathbb{R}}_{+}\right)$and $\omega \equiv 1$ near $r=0$.

Definition 3.1. Let $s \in \mathbb{N}, \gamma \in \mathbb{R}$, and $n:=\operatorname{dim} X$. We denote by $\mathcal{K}^{s, \gamma}\left(X^{\wedge}\right)$ the space of all $u(r, x) \in r^{-\frac{n}{2}} L^{2}\left(\mathbb{R}_{+} \times X, d r d x\right)$ such that

$$
\left(r \partial_{r}\right)^{k} D_{x}^{\alpha}(\omega u)(r, x) \in r^{-\frac{n}{2}} L^{2}\left(\mathbb{R}_{+} \times X, d r d x\right) \quad \text { for all } k+|\alpha| \leq s,
$$

where $D_{x}^{\alpha}$ for $\alpha=\left(\alpha_{1}, \ldots, \alpha_{n}\right)$ denotes any composition $v_{1}^{\alpha_{1}} \ldots v_{n}^{\alpha_{n}}$ of vector fields $v_{j}$ on $X$, and

$$
(1-\omega) u \in H_{\text {cone }}^{s}\left(X^{\wedge}\right),
$$

the standard Sobolev space of smoothness $s \in \mathbb{R}$ on the infinite cone $X^{\wedge}$ (cf. [12] for details).

Note that for $X=S^{n}$ (the unit sphere in $\mathbb{R}^{n+1}$ ) we have $(1-\omega) H_{\text {cone }}^{s}\left(X^{\wedge}\right)=(1-\omega) H^{s}\left(\mathbb{R}^{n+1}\right)$. The definition of $\mathcal{K}^{s, \gamma}\left(X^{\wedge}\right)$ for general $s \in \mathbb{R}$ follows by duality (with respect to the $\mathcal{K}^{0,0}$-scalar product) and interpolation. The spaces $\mathcal{K}^{s, \gamma}\left(X^{\wedge}\right)$ can be equipped with norms in terms of Hilbert space scalar products. In particular, we have

$$
\mathcal{K}^{0,0}\left(X^{\wedge}\right)=r^{-\frac{n}{2}} L^{2}\left(\mathbb{R}_{+} \times X, d r d x\right) .
$$

We shall endow each $\mathcal{K}^{s, \gamma}\left(X^{\wedge}\right)$ with the group action defined by

$$
\left(\kappa_{\lambda} u\right)(r, x)=\lambda^{\frac{n+1}{2}} u(\lambda r, x), \quad \lambda>0 .
$$

For references below we also form the Fréchet spaces

$$
\mathcal{S}^{\gamma}\left(X^{\wedge}\right)=\left\{u \in \mathcal{K}^{\infty, \gamma}\left(X^{\wedge}\right):(1-\omega) u \in \mathcal{S}\left(\overline{\mathbb{R}}_{+}, C^{\infty}(X)\right)\right\},
$$

endowed with the same group action.

REMARK 3.2. For purposes below we also consider the spaces $\mathcal{K}^{s, \gamma ; g}\left(X^{\wedge}\right):=[r]^{-g} \mathcal{K}^{s, \gamma}\left(X^{\wedge}\right), g \in \mathbb{R}$, endowed with the group action

$$
\left(\kappa_{\lambda}^{g} u\right)(r, x)=\lambda^{g+\frac{n+1}{2}} u(\lambda r, x), \quad \lambda>0 .
$$


Setting

$$
K^{s, \gamma}\left(X^{\wedge}\right)=\mathcal{K}^{s, \gamma ; s-\gamma}\left(X^{\wedge}\right),
$$

we have that $K^{s, \gamma}\left(X^{\wedge}\right)=r^{\gamma} K^{s, 0}\left(X^{\wedge}\right)$ for every $s, \gamma \in \mathbb{R}$.

Proposition 3.3. Given an edge-degenerate differential operator A, the associated operator family (3.2) represents an operator-valued symbol

$$
a(y, \eta) \in S^{\mu}\left(\Omega \times \mathbb{R}^{q} ; \mathcal{K}^{s, \gamma}\left(X^{\wedge}\right), \mathcal{K}^{s-\mu, \gamma-\mu}\left(X^{\wedge}\right)\right)
$$

for every $s, \gamma \in \mathbb{R}$. The family of operators

$$
\sigma_{\wedge}(A)(y, \eta)=r^{-\mu} \sum_{j+|\alpha| \leq \mu} a_{j \alpha}(0, y)\left(-r \partial_{r}\right)^{j}(r \eta)^{\alpha}
$$

is (twisted) homogeneous of order $\mu$, i.e., $\sigma_{\wedge}(A)(y, \lambda \eta)=\lambda^{\mu} \kappa_{\lambda} \sigma_{\wedge}(A)(y, \eta) \kappa_{\lambda}^{-1}$ for all $\lambda>0$.

REMARK 3.4. The operator family (3.2) also represents an operator-valued symbol

$$
a(y, \eta) \in S^{\mu}\left(\Omega \times \mathbb{R}^{q} ; \mathcal{K}^{s, \gamma ; g}\left(X^{\wedge}\right), \mathcal{K}^{s-\mu, \gamma-\mu ; g}\left(X^{\wedge}\right)\right)
$$

for every $s, \gamma, g \in \mathbb{R}$, where we now refer to the group action $\left\{\kappa_{\lambda}^{g}\right\}_{\lambda \in \mathbb{R}_{+}}$in both spaces, cf. Remark 3.2. In particular, we have

$$
a(y, \eta) \in S^{\mu}\left(\Omega \times \mathbb{R}^{q} ; K^{s, \gamma}\left(X^{\wedge}\right), K^{s-\mu, \gamma-\mu}\left(X^{\wedge}\right)\right)
$$

for every $s, \gamma \in \mathbb{R}$. For convenience, we shall formulate most of our results for the case $g=0$ but already mention here that they extend to the case of arbitrary weight $g \in \mathbb{R}$.

Let $\sigma_{\psi}(A)(r, x, y, \varrho, \xi, \eta)$ denote the usual homogeneous principal symbol of the operator (3.1) of order $\mu$. Together with (3.6) we then have the principal symbol of $A$,

$$
\sigma(A):=\left(\sigma_{\psi}(A), \sigma_{\wedge}(A)\right)
$$

which controls the ellipticity in the edge calculus.

The operator $A$ is called $\sigma_{\psi}$-elliptic if it is elliptic as usual and if, in addition, its rescaled symbol

$$
\widetilde{\sigma}_{\psi}(A)(x, y, \varrho, \xi, \eta):=\left.\left(r^{\mu} \sigma_{\psi}(A)\left(r, x, y, r^{-1} \varrho, \xi, r^{-1} \eta\right)\right)\right|_{r=0}
$$

does not vanish for $(\varrho, \xi, \eta) \neq 0$.

The ellipticity of $A$ with respect to both components of (3.7) also requires the bijectivity of

$$
\sigma_{\wedge}(A)(y, \eta): \mathcal{K}^{s, \gamma}\left(X^{\wedge}\right) \longrightarrow \mathcal{K}^{s-\mu, \gamma-\mu}\left(X^{\wedge}\right)
$$

for all $(y, \eta) \in \Omega \times\left(\mathbb{R}^{q} \backslash\{0\}\right)$. However, this cannot be expected to hold true without additional information. It is known that the $\sigma_{\psi}$-ellipticity of $A$ entails the Fredholm property of (3.8) for all weights $\gamma \in \mathbb{R} \backslash D$, for a discrete set $D=D(y) \subset \mathbb{R}$. The (necessary and sufficient) condition for the Fredholm property is that the subordinate principal conormal symbol

$$
\sigma_{M}(A)(y, z)=\sum_{j=0}^{\mu} a_{j 0}(0, y) z^{j}: H^{s}(X) \longrightarrow H^{s-\mu}(X)
$$

is invertible for all $z$ with $\operatorname{Re} z=\frac{n+1}{2}-\gamma$. 
Similarly as for boundary value problems, the idea of ellipticity of edge problems (in the 'usual' sense, cf. [12], [3]) is to fill up the Fredholm family (3.8) by finite rank operators to a $2 \times 2$ block matrix family of isomorphisms

$$
\begin{aligned}
& \mathcal{K}^{s, \gamma}\left(X^{\wedge}\right) \quad \mathcal{K}^{s-\mu, \gamma-\mu}\left(X^{\wedge}\right) \\
& \sigma_{\wedge}(\mathcal{A})(y, \eta): \quad \oplus \quad \longrightarrow \quad \oplus \\
& \mathbb{C}^{j_{-}} \quad \mathbb{C}^{j_{+}}
\end{aligned}
$$

with $\sigma_{\wedge}(A)(y, \eta)$ as the upper left corner, where $\mathcal{A}=\left(\begin{array}{ll}A & K \\ T & Q\end{array}\right)$ is a corresponding edge problem with the homogeneous principal edge symbol (3.9). Edge symbols are then $2 \times 2$ block matrix symbols

$$
\boldsymbol{a}(y, \eta)=\left(\begin{array}{cc}
a(y, \eta)+m(y, \eta) & 0 \\
0 & 0
\end{array}\right)+g(y, \eta) \in S^{\mu}\left(\Omega \times \mathbb{R}^{q} ; E, \widetilde{E}\right)
$$

with $E=\mathcal{K}^{s, \gamma}\left(X^{\wedge}\right) \oplus \mathbb{C}^{j_{-}}$and $\widetilde{E}=\mathcal{K}^{s-\mu, \gamma-\mu}\left(X^{\wedge}\right) \oplus \mathbb{C}^{j_{+}}$, where $g(y, \eta)$ is a so-called Green symbol and $m(y, \eta)$ a smoothing Mellin symbol, such that $\mathcal{A}=\mathrm{Op}(\boldsymbol{a})$. For details see the appendix, Section 8 .

\section{Spaces with asymptotics}

4.1. Cone Sobolev spaces. Beside the spaces $\mathcal{K}^{s, \gamma}\left(X^{\wedge}\right)$ that we defined in Definition 3.1, we are also interested in subspaces consisting of functions that have asymptotics for $r \rightarrow 0$. In this connection let

$$
P=\left\{\left(p_{j}, m_{j}, L_{j}\right): j=0, \ldots, N\right\} \text { for }\left(p_{j}, m_{j}, L_{j}\right) \in \mathbb{C} \times \mathbb{N} \times C^{\infty}(X),
$$

with $N \in \mathbb{N} \cup\{\infty\}$ and each $L_{j}$ is a finite dimensional subspace of $C^{\infty}(X)$. We also assume that $p_{k} \neq p_{l}$ for $k \neq l$ and, in case $N=\infty$, that $\operatorname{Re} p_{j} \rightarrow-\infty$ for $j \rightarrow \infty$.

Definition 4.1. Let $\gamma \in \mathbb{R}$ and $0<\theta \in \mathbb{R} \cup\{\infty\}$. A discrete asymptotic type associated with the weight data $(\gamma, \theta)$ is a set $P$ as in (4.1) that satisfies

$$
\pi_{\mathbb{C}} P:=\left\{p_{j}: j=0, \ldots, N\right\} \subset\left\{z: \frac{n+1}{2}-\gamma-\theta<\operatorname{Re} z<\frac{n+1}{2}-\gamma\right\} .
$$

The set of all such $P$ we shall denote by $\operatorname{As}(\gamma, \theta)$.

For $P \in \operatorname{As}(\gamma, \theta)$ with $\theta<\infty$ we form the space

$$
\mathcal{E}_{P}\left(X^{\wedge}\right)=\left\{u(r, x)=\omega(r) \sum_{j=0}^{N} \sum_{k=0}^{m_{j}} c_{j k}(x) r^{-p_{j}} \log ^{k} r: c_{j k} \in L_{j} \text { for all } j, k\right\},
$$

which is of finite dimension and contained in $\mathcal{S}^{\gamma}\left(X^{\wedge}\right)$. We equip $\mathcal{E}_{P}\left(X^{\wedge}\right)$ with a norm by fixing an isomorphism

$$
\begin{aligned}
\mathcal{E}_{P}\left(X^{\wedge}\right) & \cong \underbrace{L_{0} \oplus \ldots \oplus L_{0}}_{\left(m_{0}+1\right) \text {-times }} \oplus \ldots \oplus \underbrace{L_{N} \oplus \ldots \oplus L_{N}}_{\left(m_{N}+1\right) \text {-times }} \cong \mathbb{C}^{\iota(P)}, \\
\iota(P) & =\sum_{j=0}^{N}\left(m_{j}+1\right) \operatorname{dim} L_{j} .
\end{aligned}
$$

For $\theta<\infty$ and $s \in \mathbb{R}$ let us set

$$
\mathcal{K}_{\theta}^{s, \gamma}\left(X^{\wedge}\right)=\varliminf_{j \in \mathbb{N}} \mathcal{K}^{s, \gamma+\theta-\frac{1}{1+j}}\left(X^{\wedge}\right) .
$$


This Fréchet space is regarded as the subspace of functions which are flat of order $\theta$ with respect to the reference weight $\gamma$.

Definition 4.2. Let $s, \gamma \in \mathbb{R}$ and $P \in \operatorname{As}(\gamma, \theta)$ with $\theta<\infty$. Then we define

$$
\mathcal{K}_{P}^{s, \gamma}\left(X^{\wedge}\right)=\mathcal{K}_{\theta}^{s, \gamma}\left(X^{\wedge}\right)+\mathcal{E}_{P}\left(X^{\wedge}\right) .
$$

Note that the sum in the previous definition is a direct sum. In the case $P \in \operatorname{As}(\gamma, \infty)$, we set

$$
P_{k}=\left\{(p, m, L) \in P: \frac{n+1}{2}-\gamma-k<\operatorname{Re} p\right\} \in \operatorname{As}(\gamma, k), \quad k \in \mathbb{N},
$$

and then define

$$
\mathcal{K}_{P}^{s, \gamma}\left(X^{\wedge}\right)=\varliminf_{k \in \mathbb{N}} \mathcal{K}_{P_{k}}^{s, \gamma}\left(X^{\wedge}\right), \quad \mathcal{S}_{P}^{\gamma}\left(X^{\wedge}\right)=\mathcal{S}^{\gamma}\left(X^{\wedge}\right) \cap \mathcal{K}_{P}^{\infty, \gamma}\left(X^{\wedge}\right)
$$

All these spaces with asymptotics we endow with the group action $\left\{\kappa_{\lambda}\right\}_{\lambda \in \mathbb{R}_{+}}$from (3.3).

Note that in the previous constructions, we also allow $P \in \operatorname{As}(\gamma, \theta)$ to be the empty set. In this case, obviously, $\mathcal{K}_{P}^{s, \gamma}\left(X^{\wedge}\right)=\mathcal{K}_{\theta}^{s, \gamma}\left(X^{\wedge}\right)$. To unify notation, in this case we write $\mathcal{S}_{\theta}^{\gamma}\left(X^{\wedge}\right):=\mathcal{S}_{P}^{\gamma}\left(X^{\wedge}\right)$.

4.2. Edge Sobolev spaces. We shall now introduce Sobolev spaces on $\mathbb{R}^{q} \times X^{\wedge}$ that will serve as the local models for corresponding Sobolev spaces on a manifold with edge.

DeFINITION 4.3. Let $E$ be a Hilbert space with group action $\left\{\kappa_{\lambda}\right\}_{\lambda \in \mathbb{R}_{+}}$. Then the so-called abstract edge Sobolev space $\mathcal{W}^{s}\left(\mathbb{R}^{q}, E\right), s \in \mathbb{R}$, is defined as the completion of $\mathcal{S}\left(\mathbb{R}^{q}, E\right)$ with respect to the norm

$$
\|u\|_{\mathcal{W}^{s}\left(\mathbb{R}^{q}, E\right)}=\left(\int[\eta]^{2 s}\left\|\kappa_{[\eta]}^{-1} \widehat{u}(\eta)\right\|_{E}^{2} d \eta\right)^{\frac{1}{2}},
$$

where $\widehat{u}(\eta)=(F u)(\eta)$ is the Fourier transform of $u$.

The space $\mathcal{W}^{s}\left(\mathbb{R}^{q}, E\right)$ is then a Hilbert space with scalar product

$$
\langle u, v\rangle_{\mathcal{W}^{s}\left(\mathbb{R}^{q}, E\right)}=\int[\eta]^{2 s}\left\langle\kappa_{[\eta]}^{-1} \widehat{u}(\eta), \kappa_{[\eta]}^{-1} \widehat{v}(\eta)\right\rangle_{E} d \eta \text {. }
$$

In the special case of $\kappa_{\lambda}=\operatorname{id}_{E}$ for all $\lambda>0$, the edge Sobolev space $\mathcal{W}^{s}\left(\mathbb{R}^{q}, E\right)$ coincides with $H^{s}\left(\mathbb{R}^{q}, E\right)$, the usual $E$-valued Sobolev space on $\mathbb{R}^{q}$.

Note that replacing in the previous definition $[\eta]$ by $\langle\eta\rangle$ yields an equivalent norm on the space $\mathcal{W}^{s}\left(\mathbb{R}^{q}, E\right)$ and that

$$
L:=\mathcal{F}^{-1} \kappa_{[\eta]}^{-1} \mathcal{F}: \mathcal{W}^{s}\left(\mathbb{R}^{q}, E\right) \longrightarrow H^{s}\left(\mathbb{R}^{q}, E\right)
$$

is an isomorphism for each real $s$.

REMARK 4.4. If $\left\{\kappa_{\lambda}\right\}_{\lambda \in \mathbb{R}_{+}}$is a group action on $E$, there exist constants $c, M \geq 0$ such that $\left\|\kappa_{\lambda}\right\|_{\mathcal{L}(E)} \leq c \lambda^{M}$ for all $\lambda>0$. From this it immediately follows that

$$
\mathcal{W}^{s+M}\left(\mathbb{R}^{q}, E\right) \hookrightarrow H^{s}\left(\mathbb{R}^{q}, E\right) \hookrightarrow \mathcal{W}^{s-M}\left(\mathbb{R}^{q}, E\right) .
$$

Analogous constructions make sense for the case of a Fréchet space $E=\varliminf_{j \in \mathbb{N}} E^{j}$ with group action, cf. the notation after Remark 2.2. In this case we have the spaces

$$
\mathcal{W}^{s}\left(\mathbb{R}^{q}, E\right)=\lim _{j \in \mathbb{N}} \mathcal{W}^{s}\left(\mathbb{R}^{q}, E^{j}\right)
$$

and a corresponding isomorphism (4.6). 
EXAMPLE 4.5. Choosing $E=\mathcal{K}^{s, \gamma}\left(X^{\wedge}\right)$ or $E=\mathcal{K}_{P}^{s, \gamma}\left(X^{\wedge}\right)$ with the group action from (3.3), we obtain so-called weighted edge Sobolev spaces (with asymptotics)

$$
\mathcal{W}^{s, \gamma}\left(\mathbb{R}^{q} \times X^{\wedge}\right):=\mathcal{W}^{s}\left(\mathbb{R}^{q}, \mathcal{K}^{s, \gamma}\left(X^{\wedge}\right)\right), \quad \mathcal{W}_{P}^{s, \gamma}\left(\mathbb{R}^{q} \times X^{\wedge}\right):=\mathcal{W}^{s}\left(\mathbb{R}^{q}, \mathcal{K}_{P}^{s, \gamma}\left(X^{\wedge}\right)\right) .
$$

REMARK 4.6. We can also form $\mathcal{W}^{s}\left(\mathbb{R}^{q}, \mathcal{K}^{s, \gamma ; g}\left(X^{\wedge}\right)\right)$ with the spaces $\mathcal{K}^{s, \gamma ; g}\left(X^{\wedge}\right)$ and the corresponding group action from Remark 3.2. In particular, for the case $g=s-\gamma$ we obtain the spaces

$$
W^{s, \gamma}\left(\mathbb{R}^{q} \times X^{\wedge}\right):=\mathcal{W}^{s}\left(\mathbb{R}^{q}, K^{s, \gamma}\left(X^{\wedge}\right)\right) .
$$

It then can be proved (cf. [16], [6]) that, for any cut-off function $\omega(r) \in C^{\infty}\left(\overline{\mathbb{R}}_{+}\right)$,

$$
\omega W^{s, \gamma}\left(\mathbb{R}^{q} \times X^{\wedge}\right)=r^{\gamma} \omega W^{s, 0}\left(\mathbb{R}^{q} \times X^{\wedge}\right) .
$$

In our calculus we assume from now on, for convenience, that $g=0$. However, note that the (analogous) results remain true for arbitrary $g$, especially, for the case $g=s-\gamma$.

For purposes below we recall a continuity result of operators between abstract edge Sobolev spaces.

Proposition 4.7. Let $E$ and $\widetilde{E}$ be spaces endowed with group actions $\left\{\kappa_{\lambda}\right\}_{\lambda \in \mathbb{R}_{+}}$and $\left\{\tilde{\kappa}_{\lambda}\right\}_{\lambda \in \mathbb{R}_{+}}$, respectively. Moreover, let $a(y, \eta) \in S^{\mu}\left(\Omega \times \mathbb{R}^{q} ; E, \widetilde{E}\right)$ for $\Omega=\mathbb{R}^{q}$ be independent of $y$ for large $|y|$. Then

$$
\mathrm{Op}(a): \mathcal{S}\left(\mathbb{R}^{q}, E\right) \longrightarrow \mathcal{S}\left(\mathbb{R}^{q}, \widetilde{E}\right),
$$

and $\mathrm{Op}(a)$ extends for each $s \in \mathbb{R}$ to a continuous operator

$$
\mathrm{Op}(a): \mathcal{W}^{s}\left(\mathbb{R}^{q}, E\right) \longrightarrow \mathcal{W}^{s-\mu}\left(\mathbb{R}^{q}, \widetilde{E}\right) .
$$

If $E$ is the direct sum $E=E_{0}+E_{1}$ of two closed subspaces, where $\left\{\kappa_{\lambda}\right\}_{\lambda \in \mathbb{R}_{+}}$restricts to a group action on $E_{0}$ but $E_{1}$ is not necessarily invariant under the group action, we obtain by (4.6)

$$
\mathcal{W}^{s}\left(\mathbb{R}^{q}, E\right)=\mathcal{W}^{s}\left(\mathbb{R}^{q}, E_{0}\right)+\mathcal{V}^{s}\left(\mathbb{R}^{q}, E_{1}\right), \quad \mathcal{V}^{s}\left(\mathbb{R}^{q}, E_{1}\right):=L^{-1} H^{s}\left(\mathbb{R}^{q}, E_{1}\right) .
$$

This is also a direct decomposition into closed subspaces. If $E_{0}$ is orthogonal to $E_{1},(4.7)$ is also an orthogonal decomposition. In fact, if $b_{1}$ is the (orthogonal) projection in $E$ onto $E_{1}$, then

$$
P_{1}:=\mathcal{F}_{\eta \rightarrow y}^{-1}\left(\kappa_{[\eta]} b_{1} \kappa_{[\eta]}^{-1}\right) \mathcal{F}=\operatorname{Op}\left(p_{1}\right), \quad p_{1}(\eta)=\kappa_{[\eta]} b_{1} \kappa_{[\eta]}^{-1},
$$

is the (orthogonal) projection onto $\mathcal{V}^{s}\left(\mathbb{R}^{q}, E_{1}\right)$ along $\mathcal{W}^{s}\left(\mathbb{R}^{q}, E_{0}\right)$.

EXAMPLE 4.8. Let $0<\sigma<\infty$ and $S \in \operatorname{As}(\gamma-\sigma, \sigma)$ a finite asymptotic type. Then $E_{1}:=\mathcal{E}_{S}\left(X^{\wedge}\right)$ is not invariant under $\kappa_{\lambda}$ from (3.3). Taking either $E_{0}=\mathcal{K}^{s, \gamma}\left(X^{\wedge}\right)$ or $E_{0}=\mathcal{K}_{P}^{s, \gamma}\left(X^{\wedge}\right)$ and $E=E_{0}+E_{1}$, the above construction yields spaces

$$
\begin{aligned}
& \mathcal{W}^{s, \gamma}\left(\mathbb{R}^{q} \times X^{\wedge}\right)_{S}=\mathcal{W}^{s}\left(\mathbb{R}^{q}, \mathcal{K}^{s, \gamma}\left(X^{\wedge}\right)\right)+\mathcal{V}^{s}\left(\mathbb{R}^{q}, \mathcal{E}_{S}\left(X^{\wedge}\right)\right), \\
& \mathcal{W}_{P}^{s, \gamma}\left(\mathbb{R}^{q} \times X^{\wedge}\right)_{S}=\mathcal{W}^{s}\left(\mathbb{R}^{q}, \mathcal{K}_{P}^{s, \gamma}\left(X^{\wedge}\right)\right)+\mathcal{V}^{s}\left(\mathbb{R}^{q}, \mathcal{E}_{S}\left(X^{\wedge}\right)\right) .
\end{aligned}
$$

Since $\mathcal{E}_{S}\left(X^{\wedge}\right) \subset \mathcal{K}^{\infty, \gamma-\sigma}\left(X^{\wedge}\right)$, these are both subspaces of $\mathcal{W}^{s, \gamma-\sigma}\left(\mathbb{R}^{q} \times X^{\wedge}\right)$.

In the situation of Example 4.8 we shall derive in Proposition 5.11 more precise information about the structure of the corresponding projection $P_{1}$ from (4.8) and its symbol $p_{1}(\eta)$.

REMARK 4.9. In our formalism we also want to admit the choice $\sigma=0$. Then $S$ is the empty set and we simply have $\mathcal{W}_{(P)}^{s, \gamma}\left(\mathbb{R}^{q} \times X^{\wedge}\right)_{S}=\mathcal{W}_{(P)}^{s, \gamma}\left(\mathbb{R}^{q} \times X^{\wedge}\right)$. 


\section{The class of edge symbols of non-trivial type}

The aim of the present section is to introduce our new calculus with singular trace and Green operators, locally on a stretched wedge $\Omega \times X^{\wedge}$. The global situation of a manifold with edges is studied in Section 6 .

Throughout the section we fix a weight $\gamma \in \mathbb{R}$, real numbers $\mu$ and $\sigma, \tau \geq 0$, and endow $\mathbb{C}$ with the trivial group action, $\kappa_{\lambda} \equiv 1$ for all $\lambda>0$, while all spaces on $X^{\wedge}$ carry the group action from (3.3). Also, we fix asymptotic types

(5.1) $S=\left\{\left(p_{j}, m_{j}, L_{j}\right)\right\}_{j=0, \ldots, N}$

$$
\begin{aligned}
& \pi_{\mathbb{C}} S \subset\left\{z: \frac{n+1}{2}-\gamma<\operatorname{Re} z<\frac{n+1}{2}-(\gamma-\sigma)\right\}, \\
& \pi_{\mathbb{C}} T \subset\left\{z: \frac{n+1}{2}-(\gamma-\mu)<\operatorname{Re} z<\frac{n+1}{2}-(\gamma-\mu-\tau)\right\} .
\end{aligned}
$$$$
\text { (5.2) } T=\left\{\left(p_{j}^{\prime}, m_{j}^{\prime}, L_{j}^{\prime}\right)\right\}_{j=0, \ldots, N^{\prime}}
$$

We write

$$
\mathcal{K}^{s, \gamma}\left(X^{\wedge}\right)_{S}:=\mathcal{K}^{s, \gamma}\left(X^{\wedge}\right)+\mathcal{E}_{S}\left(X^{\wedge}\right)
$$

and set, for each $\eta \in \mathbb{R}^{q}$,

$$
p_{1, S}(\eta)=\kappa_{[\eta]} b_{S} \kappa_{[\eta]}^{-1}, \quad p_{0, S}(\eta)=1-p_{1, S}(\eta),
$$

with $b_{S}$ being the projection in $\mathcal{K}^{s, \gamma}\left(X^{\wedge}\right)_{S}$ on $\mathcal{E}_{S}\left(X^{\wedge}\right)$ along $\mathcal{K}^{s, \gamma}\left(X^{\wedge}\right)$, cf. (4.8). Analogous notation we use for spaces and projections associated with $T$ and $\gamma-\mu$.

Maybe it would be more precise to include in the notation of $p_{0, S}(\eta)$ and $p_{1, S}(\eta)$ the smoothnessparameter $s \in \mathbb{R}$. However, we shall not do so, since we may consider $b_{S}$ as the restriction to $\mathcal{K}^{s, \gamma}\left(X^{\wedge}\right)_{S}$ of the map $u+v \mapsto v: \mathcal{K}^{-\infty, \gamma}\left(X^{\wedge}\right) \oplus \mathcal{E}_{S}\left(X^{\wedge}\right) \rightarrow \mathcal{E}_{S}\left(X^{\wedge}\right)$. Hence $p_{j, S}(\eta)$ for small $s$ restricts to the corresponding $p_{j, S}(\eta)$ for larger $s$.

5.1. Trace and potential symbols. We begin with the intoduction and analysis of so-called singular trace symbols.

Definition 5.1. A singular trace symbol of order $\nu$ (with respect to $S$ and the weight datum $\gamma$ ) is an element

$$
t_{1}(y, \eta) \in \bigcap_{s \in \mathbb{R}} S_{\mathrm{cl}}^{\nu}\left(\Omega \times \mathbb{R}^{q} ; \mathcal{K}^{s, \gamma}\left(X^{\wedge}\right)_{S}, \mathbb{C}\right)
$$

with the property that $t_{1}(y, \eta)$ vanishes on $\mathcal{K}^{s, \gamma}\left(X^{\wedge}\right)$ for all $(y, \eta)$.

Note that when $t_{1}(y, \eta)$ vanishes on $\mathcal{K}^{s, \gamma}\left(X^{\wedge}\right)$ for all $(y, \eta)$, then also the homogeneous components $t_{1,(\nu-l)}(y, \eta)$ vanish on $\mathcal{K}^{s, \gamma}\left(X^{\wedge}\right)$ for all $l \in \mathbb{N}$. In fact, we have

$$
t_{1,(\nu)}(y, \eta)=\lim _{\lambda \rightarrow \infty} \lambda^{-\nu} t_{1}(y, \lambda \eta) \kappa_{\lambda}
$$

which yields the result for $l=0$. In a similar manner we can argue for arbitrary $l \in \mathbb{N}$.

Singular trace symbols can be characterised in a more explicit way. But before we derive this description (cf. Proposition 5.6, below), let us first illustrate how the standard trace symbols of positive type in Boutet de Monvel's algebra can be interpreted in this context.

Example 5.2. Consider the case $X^{\wedge}=\mathbb{R}_{+}$(i.e., $\left.\operatorname{dim} X=0\right)$ such that $\Omega \times \mathbb{R}_{+} \ni(y, r)$ may be interpreted as the local model of a smooth manifold with boundary. It is known that for any $\gamma>\frac{1}{2}$ with $\gamma \notin \frac{1}{2}+\mathbb{N}$ we have the identity

$$
H^{\gamma}\left(\mathbb{R}_{+}\right)=\mathcal{K}^{\gamma, \gamma}\left(\mathbb{R}_{+}\right)+\mathcal{E}_{S}\left(\mathbb{R}_{+}\right), \quad S=\left\{(j, 0): j=0, \ldots,\left[\gamma-\frac{1}{2}\right]\right\} ;
$$


here we write $[t]=\max \{m \in \mathbb{Z}: m \leq t\}$ if $t$ is a real number. $\mathcal{E}_{S}\left(\mathbb{R}_{+}\right)$consists of all finite Taylor polynomials

$$
u(r)=\omega(r) \sum_{j=0}^{\left[\gamma-\frac{1}{2}\right]} c_{j} r^{j}, \quad c_{j} \in \mathbb{C},
$$

and has dimension $\left[s-\frac{1}{2}\right]+1$. Let us now fix $0 \leq k \leq\left[\gamma-\frac{1}{2}\right]$ and define

$$
t_{1}: H^{\gamma}\left(\mathbb{R}_{+}\right) \longrightarrow \mathbb{C}, \quad t_{1} u=\left.\frac{d^{k}}{d r^{k}}\right|_{r=0} u .
$$

Obviously, $t_{1}$ vanishes on $\mathcal{K}^{\gamma, \gamma}\left(\mathbb{R}_{+}\right)$. We consider $t_{1}$ as a symbol independent of $(y, \eta)$. It then satisfies the homogeneity relation

$$
t_{1} u=\lambda^{k+\frac{1}{2}} t_{1} \kappa_{\lambda}^{-1} u \quad \text { for all } \lambda>0,
$$

which shows that $t_{1} \in S_{\mathrm{cl}}^{k+\frac{1}{2}}\left(\Omega \times \mathbb{R}^{q} ; \mathcal{K}^{\gamma, \gamma}\left(\mathbb{R}_{+}\right)_{S}, \mathbb{C}\right)$. Even more,

$$
t_{1} \in \bigcap_{s \in \mathbb{R}} S_{\mathrm{cl}}^{k+\frac{1}{2}}\left(\mathbb{R}^{q} ; \mathcal{K}^{s, \gamma}\left(\mathbb{R}_{+}\right)_{S}, \mathbb{C}\right),
$$

by extending $($ for $s<\gamma) t_{1}$ by vanishing on $\mathcal{K}^{s, \gamma}\left(\mathbb{R}_{+}\right)$. Thus we are in the above situation, here for $\sigma=\gamma$.

Let us assume for the moment that the asymptotic type $S$ only consists of a single triple $(p, m, L)$ with $p \in \mathbb{C}$ satisfying $\operatorname{Re} p<\frac{n+1}{2}-(\gamma-\sigma)$. Then $\mathcal{E}_{S}\left(X^{\wedge}\right)=\left\{\omega(r) r^{-p} \sum_{j=0}^{m} \varphi_{j}(x) \log ^{j} r: \varphi_{j} \in L\right\}$. We shall now make use of the Mellin transform. It is defined by

$$
(M v)(z)=\int_{0}^{\infty} r^{z} v(r) \frac{d r}{r}
$$

for $v \in C_{0}^{\infty}\left(\mathbb{R}_{+}\right)$and can be extended to various other spaces (for the basic properties of the Mellin transform we refer to $[\mathbf{3}]$ ). For example, if $\omega_{1}$ is an arbitrary cut-off function then

$$
M \omega_{1}: \mathcal{K}^{s, \gamma}\left(X^{\wedge}\right)_{S} \longrightarrow \mathcal{A}\left(\left\{\operatorname{Re} z>\frac{n+1}{2}-\gamma\right\} \backslash\{p\}, H^{s}(X)\right),
$$

where $\mathcal{A}(U, F)$ denotes the holomorphic functions on $U$ with values in $F$. In fact, if $u \in \mathcal{K}^{s, \gamma}\left(X^{\wedge}\right)_{S}$, then $v:=M\left(\omega_{1} u\right)$ has a pole in $p$ of multiplicity $m+1$. More precisely, if $u(r, x)=\omega(r) r^{-p} \varphi(x) \log ^{k} r$, then the principal part of $v$ is $(-1)^{k} k ! \varphi(x)(z-p)^{-(k+1)}$. This allows us to compose (5.5) with the map

$$
B_{p, k}: v \mapsto \frac{1}{2 \pi i} \int_{|z-p|<\varepsilon}(z-p)^{k} v(z) d z, \quad 0 \leq k \leq m
$$

where $\varepsilon>0$ is chosen sufficiently small. The map $B_{p, k}$ computes the Laurent coefficient of $v$ at $(z-p)^{-(k+1)}$ which belongs to the space $L$. Now the following observation is obvious:

LEMmA 5.3. Let $S$ be as in (5.1). Then any function $t(y, \eta) \in C^{\infty}\left(\Omega \times \mathbb{R}^{q}, \mathcal{L}\left(\mathcal{K}^{s, \gamma}\left(X^{\wedge}\right)_{S}, \mathbb{C}\right)\right)$ with $\left.t(y, \eta)\right|_{\mathcal{K}^{s, \gamma}\left(X^{\wedge}\right)} \equiv 0$ has a unique representation as

$$
t(y, \eta)=\sum_{j=0}^{N} \sum_{k=0}^{m_{j}} d_{j k}(y, \eta) \circ B_{p_{j}, k} \circ M \omega_{1}
$$

with $d_{j k}(y, \eta) \in C^{\infty}\left(\Omega \times \mathbb{R}^{q}, L_{j}^{*}\right)$, where $L_{j}^{*}$ denotes the dual space of $L_{j}$. In fact, given $t(y, \eta)$, then

$$
d_{j k}(y, \eta) \varphi=\frac{(-1)^{k}}{k !} t(y, \eta)\left(\omega(r) r^{-p_{j}} \varphi \log ^{k} r\right), \quad \varphi \in L_{j}
$$


Of course, the previous lemma has a corresponding formulation if we replace the parameter-space $\Omega \times \mathbb{R}^{q}$ by another one. A direct consequence of Lemma 5.3 is that singular trace symbols of order $-\infty$ associated with the asymptotic type $S$ are precisely of the form

$$
t(y, \eta)=\sum_{j=0}^{N} \sum_{k=0}^{m_{j}} d_{j k}(y, \eta)\left(\frac{1}{2 \pi i} \int_{\left|z-p_{j}\right|=\varepsilon}\left(z-p_{j}\right)^{k}\left[M\left(\omega_{1} u\right)\right](z) d z\right)
$$

with $d_{j k}(y, \eta) \in C^{\infty}(\Omega) \widehat{\otimes}_{\pi} \mathcal{S}\left(\mathbb{R}^{q}, L_{j}^{*}\right)$. Moreover, if $t_{1}(y, \eta)$ is as in Definition 5.1 and $t_{1,(\alpha)}(y, \eta)$ is a homogeneous component, then

$$
\begin{aligned}
t_{1,(\alpha)}(y, \eta) u & =|\eta|^{\alpha-\frac{n+1}{2}} \sum_{j=0}^{M} \sum_{k=0}^{m_{j}} d_{j k}^{(\alpha)}\left(y, \frac{\eta}{|\eta|}\right)\left(\frac{1}{2 \pi i} \int_{\left|z-p_{j}\right|=\varepsilon}\left(z-p_{j}\right)^{k}\left[M_{r \rightarrow z}\left(\omega_{1} u\right)\left(\frac{r}{|\eta|}\right)\right](z) d z\right) \\
& =|\eta|^{\alpha-\frac{n+1}{2}} \sum_{j=0}^{M} \sum_{k=0}^{m_{j}} d_{j k}^{(\alpha)}\left(y, \frac{\eta}{|\eta|}\right)\left(\frac{1}{2 \pi i} \int_{|z-p|=\varepsilon}|\eta|^{z}\left(z-p_{j}\right)^{k}\left[M\left(\omega_{1} u\right)\right](z) d z\right)
\end{aligned}
$$

for functions $d_{j k}^{(\alpha)}(y, \eta) \in C^{\infty}\left(\Omega \times S^{q-1}, L_{j}^{*}\right)$, where $S^{q-1}$ denotes the unit-sphere in $\mathbb{R}^{q}$. This follows by using the homogeneity relation

$$
t_{1,(\alpha)}(y, \eta)=|\eta|^{\alpha} t_{1,(\alpha)}\left(y, \frac{\eta}{|\eta|}\right) \kappa_{|\eta|}^{-1}
$$

together with the formula from Lemma 5.3 in the version for the unit-sphere. In fact, it is also true that if we define $t_{1,(\alpha)}(y, \eta)$ by (5.8) with arbitrary $d_{j k}^{(\alpha)}(y, \eta) \in C^{\infty}\left(\Omega \times S^{q-1}, L_{j}^{*}\right)$, then we obtain that $t_{1,(\alpha)}(y, \eta)$ is a smooth function on $\Omega \times\left(\mathbb{R}^{q} \backslash\{0\}\right)$ with values in $\mathcal{L}\left(\mathcal{K}^{s, \gamma}\left(X^{\wedge}\right)_{S}, \mathbb{C}\right)$ that vanishes on $\mathcal{K}^{s, \gamma}\left(X^{\wedge}\right)$ and is twisted homogeneous of order $\alpha$. The homogeneity is clear, the smoothness follows from the next result.

Lemma 5.4. Let $S$ be as in (5.1) and $t: \Omega \times\left(\mathbb{R}^{q} \backslash\{0\}\right) \rightarrow \mathcal{L}\left(\mathcal{K}^{s, \gamma}\left(X^{\wedge}\right)_{S}, \mathbb{C}\right)$ such that $\left.t(y, \eta)\right|_{\mathcal{K}^{s, \gamma}\left(X^{\wedge}\right)} \equiv$ 0 . Then $t(y, \eta)$ is a smooth function if and only if so is $s(y, \eta)=t(y, \eta) \kappa_{|\eta|}^{-1}$.

Proof. Let $b_{S}: \mathcal{K}^{s, \gamma}\left(X^{\wedge}\right)_{S} \rightarrow \mathcal{E}_{S}\left(X^{\wedge}\right)$ be the canonical projection along $\mathcal{K}^{s, \gamma}\left(X^{\wedge}\right)$. By the vanishing condition and the $\kappa_{\lambda}$-invariance of $\mathcal{K}^{s, \gamma}\left(X^{\wedge}\right)$ it is immediately seen that

$$
t(y, \eta)=t(y, \eta) \kappa_{|\eta|}^{-1} b_{S} \kappa_{|\eta|}=s(y, \eta)\left(b_{S} \kappa_{|\eta|}\right), \quad s(y, \eta)=t(y, \eta)\left(b_{S} \kappa_{|\eta|}^{-1}\right) .
$$

So it remains to show that $b_{S} \kappa_{\lambda} \in \mathcal{L}\left(\mathcal{E}_{S}\left(X^{\wedge}\right)\right)$ depends smoothly on $\lambda>0$, since from this it follows its smoothness as a function with values in $\mathcal{L}\left(\mathcal{K}^{s, \gamma}\left(X^{\wedge}\right)_{S}\right)$. However, if $u(r, x)=\omega(r) r^{-p} \varphi(x) \log ^{j} r$, then

$$
\left(b_{S} \kappa_{\lambda} u\right)(r, x)=\omega(r)(\lambda r)^{-p} \varphi(x) \log ^{j}(\lambda r)=\omega(r) r^{-p} \sum_{l=0}^{j} c_{l}(\lambda) \varphi(x) \log ^{l} r
$$

with $c_{l}(\lambda)=\left(\begin{array}{l}j \\ l\end{array}\right) \log ^{j-l} \lambda$. This at once implies the requested smoothness.

There is an obvious analogous version of Lemma 5.4 by replacing $\Omega \times\left(\mathbb{R}^{q} \backslash\{0\}\right)$ by $\Omega \times \mathbb{R}^{q}$ and $\kappa_{|\eta|}$ by $\kappa_{[\eta]}$ or $\kappa_{\langle\eta\rangle}$.

Lemma 5.5. Let $S$ be as in (5.1) and $t \in \bigcap_{s \in \mathbb{R}} \mathcal{L}\left(\mathcal{K}^{s, \gamma}\left(X^{\wedge}\right)_{S}, \mathbb{C}\right)$ such that $t$ vanishes on $\mathcal{K}^{s, \gamma}\left(X^{\wedge}\right)$ for each $s$. Then

$$
c_{\varepsilon}^{-1}\langle\eta\rangle^{-\varepsilon}\|t\|_{\mathcal{L}\left(\mathcal{K}^{s, \gamma}\left(X^{\wedge}\right)_{S}, \mathbb{C}\right)} \leq\langle\eta\rangle^{\frac{n+1}{2}-\gamma+\sigma}\left\|t \kappa_{\langle\eta\rangle}\right\|_{\mathcal{L}\left(\mathcal{K}^{s, \gamma}\left(X^{\wedge}\right)_{S}, \mathbb{C}\right)} \leq c_{\varepsilon}\langle\eta\rangle^{\varepsilon}\|t\|_{\mathcal{L}\left(\mathcal{K}^{s, \gamma}\left(X^{\wedge}\right)_{S}, \mathbb{C}\right)}
$$

with both $\varepsilon>0$ and $c_{\varepsilon} \geq 1$ neither depending on $s \in \mathbb{R}, \eta \in \mathbb{R}^{q}$ nor on $t$ with the required properties. 
ProOF. Arguing as in the proof of Lemma 5.4, we get

$$
\|t\|_{\mathcal{L}\left(\mathcal{K}^{s, \gamma}\left(X^{\wedge}\right)_{S}, \mathbb{C}\right)} \leq\left\|t \kappa_{\langle\eta\rangle}\right\|_{\mathcal{L}\left(\mathcal{K}^{s, \gamma}\left(X^{\wedge}\right)_{S}\right)}\left\|b_{S} \kappa_{\langle\eta\rangle}^{-1}\right\|_{\mathcal{L}\left(\mathcal{E}_{S}\left(X^{\wedge}\right)\right)}
$$

and $\left\|t \kappa_{\langle\eta\rangle}\right\|_{\mathcal{L}\left(\mathcal{K}^{s, \gamma}\left(X^{\wedge}\right)_{S}, \mathbb{C}\right)} \leq\|t\|_{\mathcal{L}\left(\mathcal{K}^{s, \gamma}\left(X^{\wedge}\right)_{S}, \mathbb{C}\right)}\left\|b_{S} \kappa_{\langle\eta\rangle}\right\|_{\mathcal{L}\left(\mathcal{E}_{S}\left(X^{\wedge}\right)\right)}$. For the norm estimate of $b_{S} \kappa_{\lambda}$ we have to consider terms as in (5.9) and to note that

$$
\left|\lambda^{-p} \log ^{k} \lambda\right| \leq c_{k, \varepsilon} \max \left(\lambda^{\varepsilon}, \lambda^{-\varepsilon}\right) \lambda^{-\operatorname{Re} p} \quad \text { for all } \lambda>0,
$$

where we choose $\varepsilon$ so small that $\operatorname{Re} p+\varepsilon \leq \frac{n+1}{2}-\gamma+\sigma$.

The above observations now lead to the announced description of singular trace symbols. For this, let $\chi(\eta)$ be a zero excision function on $\mathbb{R}^{q}$.

Proposition 5.6. A function $t_{1}(y, \eta) \in C^{\infty}\left(\Omega \times \mathbb{R}^{q}, \mathcal{L}\left(\mathcal{K}^{s, \gamma}\left(X^{\wedge}\right)_{S}, \mathbb{C}\right)\right)$ is a singular trace symbol of order $\nu$ in the sense of Definition 5.1, if and only if there exists a sequence of homogeneous components $t_{1,(\nu-l)}, l \in \mathbb{N}$, of the form (5.8) (with $\alpha$ replaced by $\nu-l$ ) such that for each given $M \in \mathbb{N}$ there exists an $L \in \mathbb{N}$ such that the difference $t_{1}(y, \eta)-\sum_{l=0}^{L} \chi(\eta) t_{1,(\nu-l)}(y, \eta)$ is of the form (5.7) with all $d_{j k}(y, \eta)$ belonging to $S^{-M}\left(\Omega \times \mathbb{R}^{q}\right)$.

Before defining our new class of trace symbols let us recall the structure of a trace symbol from the standard edge algebra: It is a symbol

$$
t_{0}(y, \eta) \in \bigcap_{s \in \mathbb{R}} S_{\mathrm{cl}}^{\nu}\left(\Omega \times \mathbb{R}^{q} ; \mathcal{K}^{s, \gamma}\left(X^{\wedge}\right), \mathbb{C}\right),
$$

such that the (pointwise) formal adjoint symbol satisfies, for some $\varepsilon>0$,

$$
t_{0}(y, \eta)^{*} \in S_{\mathrm{cl}}^{\nu}\left(\Omega \times \mathbb{R}^{q} ; \mathbb{C}, \mathcal{S}_{\varepsilon}^{-\gamma}\left(X^{\wedge}\right)\right) .
$$

Definition 5.7. A trace symbol of order $\nu$ in the local edge calculus on $X^{\wedge} \times \Omega$ (with respect to $S$ and the weight-datum $\gamma)$ is an operator family $t(y, \eta)$ such that

$$
t(y, \eta) \in \bigcap_{s \in \mathbb{R}} S_{\mathrm{cl}}^{\nu}\left(\Omega \times \mathbb{R}^{q} ; \mathcal{K}^{s, \gamma}\left(X^{\wedge}\right)_{S}, \mathbb{C}\right),
$$

and $t(y, \eta)$ has a representation

$$
t(y, \eta)=t_{0}(y, \eta) p_{0, S}(\eta)+t_{1}(y, \eta)
$$

with a $\nu$-th order singular trace symbol $t_{1}(y, \eta)$ and a $t_{0}(y, \eta)$ being a trace symbol in the standard edge symbol algebra as described above. If $t_{1}(y, \eta) \equiv 0$, we call $t(y, \eta)$ also a regular trace symbol.

TheOREm 5.8. Let $t(y, \eta)$ be a trace symbol as in Definition 5.7 for $\Omega=\mathbb{R}^{q}$, and let $t(y, \eta)$ be independent of $y$ for large $|y|$. Then $\mathrm{Op}(t)$ induces continuous operators

$$
\mathrm{Op}(t): \mathcal{W}^{s}\left(\mathbb{R}^{q}, \mathcal{K}^{s, \gamma}\left(X^{\wedge}\right)_{S}\right) \rightarrow H^{s-\nu}\left(\mathbb{R}^{q}\right), \quad s \in \mathbb{R},
$$

and we have

$$
\mathrm{Op}\left(t_{1}\right) u=0 \quad \text { for all } u \in \mathcal{W}^{s}\left(\mathbb{R}^{q}, \mathcal{K}^{s, \gamma}\left(X^{\wedge}\right)\right) .
$$

Moreover, the singular trace operators are localised at the edges (in contrast to the regular trace operators $)$, i.e., if $v \in \mathcal{W}^{s}\left(\Omega, \mathcal{K}^{s, \gamma}\left(X^{\wedge}\right)_{S}\right)$ vanishes in a neighbourhood of $r=0$ then $\operatorname{Op}\left(t_{1}\right) v=0$. 
Proof. Property (5.10) follows from Proposition 5.11 (see below) and Proposition 4.7, noting that $\mathcal{W}^{s}\left(\mathbb{R}^{q}, \mathbb{C}\right)=H^{s}\left(\mathbb{R}^{q}\right)$. The relation (5.11) holds true, since $t_{1}(y, \eta) \widehat{u}(\eta) \equiv 0$ for any $u \in$ $\mathcal{S}\left(\mathbb{R}^{q}, \mathcal{K}^{s, \gamma}\left(X^{\wedge}\right)\right)$. For the latter statement note that the vanishing of $v$ near $r=0$ implies that, in fact, $v \in \mathcal{W}^{s}\left(\Omega, \mathcal{K}^{s, \gamma}\left(X^{\wedge}\right)\right)$. Then apply (5.11).

We finish this section by defining potential symbols. For their definition it will be convenient to use the notation

$$
\mathcal{S}_{\varepsilon}^{\gamma-\mu}\left(X^{\wedge}\right)_{T}=\mathcal{S}_{\varepsilon}^{\gamma-\mu}\left(X^{\wedge}\right) \oplus \mathcal{E}_{T}\left(X^{\wedge}\right), \quad \varepsilon>0
$$

Definition 5.9. A potential symbol of order $\nu$ (with respect to $T$ and the weight-datum $\gamma-\mu)$ is a symbol

$$
k(y, \eta) \in S_{\mathrm{cl}}^{\nu}\left(\Omega \times \mathbb{R}^{q} ; \mathbb{C}, \mathcal{S}_{\varepsilon}^{\gamma-\mu}\left(X^{\wedge}\right)_{T}\right)
$$

for some $\varepsilon=\varepsilon(k)>0$.

If we write $k(y, \eta)=p_{0, T}(\eta) k(y, \eta)+p_{1, T}(\eta) k(y, \eta)$ then the first summand is a potential symbol from the usual edge-algebra associated with the weight-datum $\gamma-\mu$.

Also potential symbols can be characterized in a more explicit way. In fact, symbols (5.13) are precisely those of the form

$$
\mathbb{C} \ni c \mapsto[\eta]^{\frac{n+1}{2}} \widetilde{k}(y, \eta ; r[\eta], x) c \in \mathcal{S}_{\varepsilon}^{\gamma-\mu}\left(X^{\wedge}\right)_{T}
$$

with a symbol kernel

$$
\widetilde{k}(y, \eta ; r, x) \in S_{\mathrm{cl}}^{\nu}\left(\Omega \times \mathbb{R}^{q}\right) \widehat{\otimes}_{\pi} \mathcal{S}_{\varepsilon}^{\gamma-\mu}\left(X_{(r, x)}^{\wedge}\right)_{T} .
$$

5.2. Parameter-dependent families of projections. We now investigate more deeply the pseudodifferential structure of the family of projections $p_{1, S}(\eta)$, cf. (5.3).

Let us define, for $0 \leq j \leq N$ and $0 \leq k \leq m_{j}$, zero order symbols by

$$
t_{j k}(\eta) u=[\eta]^{-\frac{n+1}{2}} \frac{1}{2 \pi i} \int_{\left|z-p_{j}\right|=\varepsilon}\left(z-p_{j}\right)^{k} M\left(\omega_{1} u(r /[\eta])\right)(z) d z .
$$

Using the unitary isomorphism $\mathcal{E}_{S}\left(X^{\wedge}\right) \cong \mathbb{C}^{\iota(S)}$, see (4.3), this gives rise to a map

$$
t(\eta)=t_{S}(\eta):=\left(t_{j k}(\eta)\right)_{\substack{0 \leq j \leq N, 0 \leq k \leq m_{j}}}: \mathcal{K}^{s, \gamma}\left(X^{\wedge}\right)_{S} \longrightarrow \mathbb{C}^{\iota(S)} .
$$

We have $t(\lambda \eta)=t(\eta) \kappa_{\lambda}^{-1}$ for $\lambda \geq 1,|\eta| \geq 1$, hence $t(\eta) \in S_{\mathrm{cl}}^{0}\left(\mathbb{R}^{q} ; \mathcal{K}^{s, \gamma}\left(X^{\wedge}\right)_{S}, \mathbb{C}^{\iota(S)}\right)$ (in fact, each component of $t(\eta)$ is a singular trace symbol). Moreover, let us define a (system of) potential symbols

by setting, for each $c=\left(c_{j k}\right)_{\substack{0 \leq j \leq N \\ 0 \leq k \leq m_{j}}} \in \mathbb{C}^{\iota(S)}$,

$$
k(\eta)=k_{S}(\eta): \mathbb{C}^{\iota(S)} \longrightarrow \mathcal{K}^{s, \gamma}\left(X^{\wedge}\right)_{S}
$$

$$
k(\eta) c=[\eta]^{\frac{n+1}{2}} \omega(r[\eta]) \sum_{j=0}^{N} \sum_{k=0}^{m_{j}} c_{j k}(r[\eta])^{-p_{j}} \log ^{k}(r[\eta])
$$

(on the right-hand side we have identified $c_{j k}$ with an element in $L_{j}$, using the identification (4.3)). We then have $k(\lambda \eta)=\kappa_{\lambda} k(\eta)$ for $\lambda \geq 1,|\eta| \geq 1$ which entails $k(\eta) \in S_{\mathrm{cl}}^{0}\left(\mathbb{R}^{q} ; \mathbb{C}^{\iota(S)}, \mathcal{K}^{s, \gamma}\left(X^{\wedge}\right)_{S}\right)$. From the construction if follows that $t(\eta) k(\eta)=\operatorname{id}_{\mathbb{C}^{\iota}(S)}$ for all $\eta \in \mathbb{R}^{q}$, and hence

$$
k(\eta) t(\eta): \mathcal{K}^{s, \gamma}\left(X^{\wedge}\right)_{S} \longrightarrow \mathcal{K}^{s, \gamma}\left(X^{\wedge}\right)_{S}
$$


is a family of continuous projections.

LEMma 5.10. If $k(\eta)$ and $t(\eta)$ are constructed as above, then

$$
p_{0, S}(\eta)=1-k(\eta) t(\eta) \quad \text { for all } \eta \in \mathbb{R}^{q} .
$$

Proof. By conjugation with $\kappa_{[\eta]}$ the claim is equivalent to showing that

$$
b_{S}=\left(\kappa_{[\eta]}^{-1} k(\eta)\right)\left(t(\eta) \kappa_{[\eta]}\right)
$$

for each $\eta$. However, this is true, since the first factor on the right-hand side is the map

$$
c=\left(c_{j k}\right) \mapsto \omega(r) \sum_{j=0}^{N} \sum_{k=0}^{m_{j}} c_{j k} r^{-p_{j}} \log ^{k} r,
$$

while the second factor equals $\left(t_{j k}\right)$ with $t_{j k} u=\frac{1}{2 \pi i} \int_{\left|z-p_{j}\right|=\varepsilon}\left(z-p_{j}\right)^{k} M\left(\omega_{1} u\right)(z) d z$.

By straightforward calculation, for any $u \in \mathcal{K}^{s, \gamma}\left(X^{\wedge}\right)$,

$$
p_{0, S}(\eta)\left(u+\omega(r) \sum_{j=0}^{N} \sum_{k=0}^{m_{j}} c_{j k} r^{-p_{j}} \log ^{k} r\right)=u+(\omega(r)-\omega(r[\eta])) \sum_{j=0}^{M} \sum_{k=0}^{m_{j}} c_{j k} r^{-p_{j}} \log ^{k} r .
$$

From this formula and by Lemma 5.10 we deduce the following:

Proposition 5.11. Let $s \in \mathbb{R}$. Then $p_{0, S}(\eta) \in S_{\mathrm{cl}}^{0}\left(\mathbb{R}^{q} ; \mathcal{K}^{s, \gamma}\left(X^{\wedge}\right)_{S}, \mathcal{K}^{s, \gamma}\left(X^{\wedge}\right)\right)$ and

$$
\mathrm{Op}\left(p_{0, S}\right): \mathcal{W}^{s}\left(\mathbb{R}^{q}, \mathcal{K}^{s, \gamma}\left(X^{\wedge}\right)_{S}\right) \longrightarrow \mathcal{W}^{s}\left(\mathbb{R}^{q}, \mathcal{K}^{s, \gamma}\left(X^{\wedge}\right)_{S}\right)
$$

is the projection onto $\mathcal{W}^{s}\left(\mathbb{R}^{q}, \mathcal{K}^{s, \gamma}\left(X^{\wedge}\right)\right)$ along $\mathcal{V}^{s}\left(\mathbb{R}^{q}, \mathcal{E}_{S}\left(X^{\wedge}\right)\right)$. Moreover,

$$
D_{\eta}^{\alpha} p_{0, S}(\eta) \in S_{\mathrm{cl}}^{-|\alpha|}\left(\mathbb{R}^{q} ; \mathcal{K}^{s, \gamma}\left(X^{\wedge}\right)_{S}, \mathcal{S}^{\infty}\left(X^{\wedge}\right)\right) \quad \text { for all }|\alpha| \geq 1
$$

(cf. (3.4)), and $D_{\eta}^{\alpha} p_{0, S}(\eta)$ vanishes on $\mathcal{K}^{s, \gamma}\left(X^{\wedge}\right)$ for each $\eta$.

In the terminology introduced in the next section, (5.17) means that $D_{\eta}^{\alpha} p_{0, S}(\eta)$ is a singular Green symbol of order $-|\alpha|$ whenever $|\alpha| \geq 1$.

5.3. Regular and singular Green symbols. Besides trace and potential symbols there is another category of so-called Green symbols needed for our new calculus. They are build up from regular and singular Green symbols.

Definition 5.12. A singular Green symbol of order $\nu$ (with respect to $S, T$, and the weight-datum $(\gamma, \gamma-\mu, \theta))$ is a symbol

$$
g_{1}(y, \eta) \in \bigcap_{s \in \mathbb{R}} S_{\mathrm{cl}}^{\nu}\left(\Omega \times \mathbb{R}^{q} ; \mathcal{K}^{s, \gamma}\left(X^{\wedge}\right)_{S}, \mathcal{S}_{\varepsilon}^{\gamma-\mu}\left(X^{\wedge}\right)_{T}\right)
$$

(see (5.12) for the notation) that vanishes on $\mathcal{K}^{s, \gamma}\left(X^{\wedge}\right)$ for all $(y, \eta)$, and where $\varepsilon=\varepsilon\left(g_{1}\right)>0$.

Along the lines of the material from Section 5.1, singular Green symbols have a more explicit representation: If $g_{1}(y, \eta)$ is as in Definition 5.12, all homogeneous components $g_{1,(\nu-j)}, j \in \mathbb{N}$, pointwise vanish on $\mathcal{K}^{s, \gamma}\left(X^{\wedge}\right)$. If $S$ is as in (5.1) then

$$
\begin{aligned}
g_{1,(\nu-j)}(y, \eta) & =|\eta|^{\nu-j} \sum_{j=0}^{N} \sum_{k=0}^{m_{j}} \kappa_{|\eta|} \circ e_{j k}\left(y, \frac{\eta}{|\eta|}\right) \circ B_{p_{j}, k} \circ M_{\gamma-\sigma-\frac{n}{2}} \omega_{1} \circ \kappa_{|\eta|}^{-1} \\
& : \mathcal{K}^{s, \gamma}\left(X^{\wedge}\right)_{S} \rightarrow \mathcal{S}_{\varepsilon}^{\gamma-\mu}\left(X^{\wedge}\right)_{T}
\end{aligned}
$$


where $B_{p, k}$ is as in the formula (5.6) and with uniquely determined functions

$$
e_{j k}(y, \eta) \in C^{\infty}\left(\Omega \times S^{q-1}, \mathcal{L}\left(L_{j}, \mathcal{S}_{\varepsilon}^{\gamma-\mu}\left(X^{\wedge}\right)_{T}\right)\right) .
$$

Identifying $e_{j k}(y, \eta)$ with an $L_{j}^{*}$-valued function

$$
l_{j k}^{*}(y, \eta ; r, x) \in C^{\infty}\left(\Omega \times S^{q-1}\right) \widehat{\otimes}_{\pi} \mathcal{S}_{\varepsilon}^{\gamma-\mu}\left(X^{\wedge}\right)_{T} \widehat{\otimes}_{\pi} L_{j}^{*},
$$

we obtain

$$
\begin{aligned}
& {\left[g_{1,(\nu-j)}(y, \eta) u\right](r, x)=} \\
& \quad|\eta|^{\frac{n+1}{2}+\nu-j} \sum_{j=0}^{N} \sum_{k=0}^{m_{j}} l_{j k}^{*}\left(y, \frac{\eta}{|\eta|} ; r|\eta|, x\right)\left(\frac{1}{2 \pi i} \int_{\left|z-p_{j}\right|=\varepsilon}\left(z-p_{j}\right)^{k}\left[M_{r^{\prime} \rightarrow z}\left(\kappa_{|\eta|}^{-1} \omega_{1} u\right)\right](z) d z\right) .
\end{aligned}
$$

In other words, each homogeneous component of a singular Green symbol has a unique representation as a finite linear combination of summands that are the (pointwise) composition of homogeneous components of a singular trace symbol and a potential symbol.

Definition 5.13. A Green symbol of order $\nu$ (with respect to $S, T$, and the weight-datum $(\gamma, \gamma-$ $\mu, \theta)$ ) in the local edge calculus on $X^{\wedge} \times \Omega$ is any operator function

$$
g(y, \eta) \in \bigcap_{s \in \mathbb{R}} S_{\mathrm{cl}}^{\nu}\left(\Omega \times \mathbb{R}^{q} ; \mathcal{K}^{s, \gamma}\left(X^{\wedge}\right)_{S}, \mathcal{K}^{\infty, \gamma-\mu}\left(X^{\wedge}\right)_{T}\right)
$$

which has a representation

$$
g(y, \eta)=g_{0}(y, \eta) p_{0, S}(\eta)+g_{1}(y, \eta)
$$

where $g_{1}(y, \eta)$ is a singular Green symbol and, for some $\varepsilon=\varepsilon\left(g_{0}\right)>0$,

$$
\begin{gathered}
g_{0}(y, \eta) \in \bigcap_{s \in \mathbb{R}} S_{\mathrm{cl}}^{\nu}\left(\Omega \times \mathbb{R}^{q} ; \mathcal{K}^{s, \gamma}\left(X^{\wedge}\right), \mathcal{S}_{\varepsilon}^{\gamma-\mu}\left(X^{\wedge}\right)_{T}\right), \\
g_{0}(y, \eta)^{*} \in \bigcap_{s \in \mathbb{R}} S_{\mathrm{cl}}^{\nu}\left(\Omega \times \mathbb{R}^{q} ; \mathcal{K}^{s,-\gamma+\mu+\tau}\left(X^{\wedge}\right), \mathcal{S}_{\varepsilon}^{-\gamma}\left(X^{\wedge}\right)\right),
\end{gathered}
$$

where $^{*}$ refers to the pointwise adjoint with respect to the $\mathcal{K}^{0,0}\left(X^{\wedge}\right)$ scalar product. If $g_{1}(y, \eta) \equiv 0$, we call $g(y, \eta)$ a regular Green symbol.

Note that if $g_{0}(y, \eta)$ is as in the previous definition, then

$$
p_{0, T}(\eta) g_{0}(y, \eta) \in \mathcal{R}_{G}^{\nu}\left(\Omega \times \mathbb{R}^{q} ;(\gamma, \gamma-\mu)\right)
$$

is a Green symbol from the standard edge algebra, cf. Section 8.

EXAMPLE 5.14. The pointwise composition

$$
g(y, \eta):=k(y, \eta) t(y, \eta)
$$

of a $\nu_{0}$-th order trace symbol and a $\nu_{1}$-th order potential symbol in the sense of Definitions 5.7 and 5.9 , respectively, is a Green symbol of order $\nu=\nu_{0}+\nu_{1}$ in the sense of the Definition 5.13.

We shall also condsider vector-valued variants of trace, potential, and Green symbols, i.e.

$$
\begin{aligned}
t(y, \eta) & : \mathcal{K}^{s, \gamma}\left(X^{\wedge}, \mathbb{C}^{k}\right) \longrightarrow \mathbb{C}^{j_{+}} \\
k(y, \eta) & : \mathbb{C}^{j_{-}} \longrightarrow \mathcal{K}^{s-\mu, \gamma-\mu}\left(X^{\wedge}, \mathbb{C}^{l}\right)_{T}, \\
g(y, \eta) & : \mathcal{K}^{s, \gamma}\left(X^{\wedge}, \mathbb{C}^{k}\right)_{S} \longrightarrow \mathcal{K}^{s-\mu, \gamma-\mu}\left(X^{\wedge}, \mathbb{C}^{l}\right)_{T}
\end{aligned}
$$


where $\mathcal{K}^{s, \gamma}\left(X^{\wedge}, \mathbb{C}^{k}\right)=\underset{j=1}{\bigoplus} \mathcal{K}^{s, \gamma}\left(X^{\wedge}\right)$, and analogously for the other spaces. This is achieved by considering matrices of corresponding size, whose entries are all trace, potential, or Green symbols of same order and with respect to the same data.

Definition 5.15. Let $1 \leq k, l \in \mathbb{N}$ and $j_{+}, j_{-} \in \mathbb{N}$ (possibly also 0 ). Then

$$
\mathcal{R}_{G}^{\nu}\left(\Omega \times \mathbb{R}^{q} ;(\gamma, \gamma-\mu),\left(k, l ; j_{-}, j_{+}\right)\right)_{S, T}
$$

denotes the space of all $(2 \times 2)$-block matrix symbols

$$
g(y, \eta)=\left(\begin{array}{ll}
g_{11}(y, \eta) & g_{12}(y, \eta) \\
g_{21}(y, \eta) & g_{22}(y, \eta)
\end{array}\right): \begin{array}{cccc}
\mathcal{K}^{s, \gamma}\left(X^{\wedge}, \mathbb{C}^{k}\right)_{S} & & \mathcal{K}^{s-\mu, \gamma-\mu}\left(X^{\wedge}, \mathbb{C}^{l}\right)_{T} \\
\oplus & \mathbb{C}^{j_{-}} & \longrightarrow & \oplus \\
\mathbb{C}^{j_{+}}
\end{array},
$$

where $g_{11}(y, \eta)$ is an $(l \times k)$-matrix of $\nu$-th order Green symbols, $g_{21}(y, \eta)$ is an $\left(j_{+} \times k\right)$-matrix of $\nu$-th order trace symbols, $g_{12}(y, \eta)$ is an $\left(j_{+} \times l\right)$-matrix of $\nu$-th order potential symbols (all with respect to $S, T$ and the weight-datum $(\gamma, \gamma-\mu)$, and $g_{22}(y, \eta)$ is an $\left(j_{+} \times j_{-}\right)$-matrix of scalar symbols from $S_{\mathrm{cl}}^{\nu}\left(\Omega \times \mathbb{R}^{q}\right)$.

To have a convenient terminology at hand, we shall call symbols (5.20) again Green symbols. Note that they are particular operator-valued symbols

$$
g(y, \eta) \in S_{\mathrm{cl}}^{\nu}\left(\Omega \times \mathbb{R}^{q} ; \mathcal{K}^{s, \gamma}\left(X^{\wedge}, \mathbb{C}^{k}\right)_{S} \oplus \mathbb{C}^{j-}, \mathcal{S}^{\gamma-\mu}\left(X^{\wedge}, \mathbb{C}^{l}\right)_{T} \oplus \mathbb{C}^{j_{+}}\right) .
$$

5.4. The full symbol class and its calculus. For the following considerations recall that $\mathcal{K}^{s-\mu, \gamma-\mu}\left(X^{\wedge}\right)$ is a subspace of $\mathcal{K}^{s-\mu, \gamma-\mu}\left(X^{\wedge}\right)_{T}$.

Definition 5.16. Let $\nu \in \mathbb{R}$ with $\mu-\nu \in \mathbb{N}$. The space

$$
\mathcal{R}^{\nu}\left(\Omega \times \mathbb{R}^{q} ;(\gamma, \gamma-\mu),\left(k, l ; j_{-}, j_{+}\right)\right)_{S, T}
$$

consists of all symbols $\boldsymbol{a}(y, \eta)$ of the form

$$
\boldsymbol{a}(y, \eta)=\left(\begin{array}{cc}
a_{0}(y, \eta) p_{0, S}(\eta) & 0 \\
0 & 0
\end{array}\right)+g(y, \eta): \begin{array}{ccc}
\mathcal{K}^{s, \gamma}\left(X^{\wedge}, \mathbb{C}^{k}\right)_{S} & \mathcal{K}^{s-\mu, \gamma-\mu}\left(X^{\wedge}, \mathbb{C}^{l}\right)_{T} \\
\mathbb{C}^{j_{-}} & \longrightarrow & \oplus \\
\mathbb{C}^{j_{+}}
\end{array}
$$

with an arbitrary standard edge symbol $a_{0}(y, \eta) \in \mathcal{R}^{\nu}\left(\Omega \times \mathbb{R}^{q} ;(\gamma, \gamma-\mu),(k, l)\right)($ cf. Section 8$)$, and some Green symbol $g(y, \eta) \in \mathcal{R}_{G}^{\nu}\left(\Omega \times \mathbb{R}^{q} ;(\gamma, \gamma-\mu),\left(k, l, j_{-}, j_{+}\right)\right)_{S, T}$.

Observe that then, for every $s \in \mathbb{R}$,

$$
\begin{aligned}
& \mathcal{R}^{\nu}\left(\Omega \times \mathbb{R}^{q} ;(\gamma, \gamma-\mu),\left(k, l ; j_{-}, j_{+}\right)\right)_{S, T} \subset \\
& \quad \subset S^{\nu}\left(\Omega \times \mathbb{R}^{q} ; \mathcal{K}^{s, \gamma}\left(X^{\wedge}, \mathbb{C}^{k}\right)_{S} \oplus \mathbb{C}^{j_{-}}, \mathcal{K}^{s-\nu, \gamma-\mu}\left(X^{\wedge}, \mathbb{C}^{l}\right)_{T} \oplus \mathbb{C}^{j_{+}}\right) .
\end{aligned}
$$

Thus if $\Omega=\mathbb{R}^{q}$ and $\boldsymbol{a}(y, \eta)$ as in (5.22) is independent of $y$ for large $|y|$, then it induces continuous operators

$$
\begin{aligned}
& \mathcal{W}^{s}\left(\mathbb{R}^{q}, \mathcal{K}^{s, \gamma}\left(X^{\wedge}, \mathbb{C}^{k}\right)_{S}\right) \quad \mathcal{W}^{s-\nu}\left(\mathbb{R}^{q}, \mathcal{K}^{s-\nu, \gamma-\mu}\left(X^{\wedge}, \mathbb{C}^{l}\right)_{T}\right) \\
& \begin{array}{ccccc}
\mathrm{Op}(\boldsymbol{a}): & \oplus & \rightarrow & \oplus
\end{array}
\end{aligned}
$$


Definition 5.17. For $\boldsymbol{a}(y, \eta) \in \mathcal{R}^{\mu}\left(\Omega \times \mathbb{R}^{q} ;(\gamma, \gamma-\mu),\left(k, l ; j_{-}, j_{+}\right)\right)_{S, T}$ we set

$$
\sigma_{\wedge}(\boldsymbol{a})(y, \eta)=\left(\begin{array}{cc}
\sigma_{\wedge}\left(a_{0}\right)(y, \eta) \sigma_{\wedge}\left(p_{0, S}\right)(\eta) & 0 \\
0 & 0
\end{array}\right)+\sigma_{\wedge}(g)(y, \eta),
$$

where $\sigma_{\wedge}\left(a_{0}\right)$ is the standard principal edge symbol (cf. Section 8) and $\sigma_{\wedge}\left(p_{0, S}\right)(\eta):=1-\kappa_{|\eta|} b_{S} \kappa_{|\eta|}^{-1}$. Moreover, $\sigma_{\wedge}(g)(y, \eta)$ denotes the homogeneous principal symbol of $g(y, \eta)$ as a classical symbol of order $\mu$, cf. (5.21). We call $\sigma_{\wedge}(\boldsymbol{a})(y, \eta)$ the principal edge symbol of $\boldsymbol{a}(y, \eta)$.

From this definition if follows that

$$
\sigma_{\wedge}(\boldsymbol{a})(y, \lambda \eta)=\lambda^{\mu} \kappa_{\lambda} \sigma_{\wedge}(\boldsymbol{a})(y, \eta) \kappa_{\lambda}^{-1}
$$

as a family of operators $\mathcal{K}^{s, \gamma}\left(X^{\wedge}, \mathbb{C}^{k}\right)_{S} \rightarrow \mathcal{K}^{s-\mu, \gamma-\mu}\left(X^{\wedge}, \mathbb{C}^{l}\right)_{T}$ with the usual group action defined by $\left(\kappa_{\lambda} u\right)(r, x)=\lambda^{\frac{n+1}{2}} u(\lambda r, x)$ for $\lambda \in \mathbb{R}_{+}$.

Definition 5.18. Let $\boldsymbol{a}(y, \eta) \in \mathcal{R}^{\mu}\left(\Omega \times \mathbb{R}^{q} ;(\gamma, \gamma-\mu),\left(k, l ; j_{-}, j_{+}\right)\right)_{S, T}$ be as in $(5.22)($ with $\nu=\mu)$. Then we define the conormal symbol of $\boldsymbol{a}$ as

$$
\sigma_{M}(\boldsymbol{a})(y, z)=\sigma_{M}\left(a_{0}\right)(y, z),
$$

where $\sigma_{M}\left(a_{0}\right)$ denotes the standard conormal symbol, cf. Section 8.

Proposition 5.19. If $\boldsymbol{a}(y, \eta) \in \mathcal{R}^{\nu}\left(\Omega \times \mathbb{R}^{q} ;(\gamma, \gamma-\mu),\left(k, l ; j_{-}, j_{+}\right)\right)_{S, T}$ then

$$
\partial_{\eta}^{\alpha} \partial_{y}^{\beta} \boldsymbol{a}(y, \eta) \in \mathcal{R}^{\nu-|\alpha|}\left(\Omega \times \mathbb{R}^{q} ;(\gamma, \gamma-\mu),\left(k, l ; j_{-}, j_{+}\right)\right)_{S, T}
$$

for any multi-indices $\alpha, \beta \in \mathbb{N}^{q}$.

THEOREM 5.20. For $j \in \mathbb{N}$ let $\boldsymbol{a}_{j}(y, \eta) \in \mathcal{R}^{\nu-k}\left(\Omega \times \mathbb{R}^{q} ;(\gamma, \gamma-\mu),\left(k, l ; j_{-}, j_{+}\right)\right)_{S, T}$ such that the $\varepsilon$-weights involved in the Green symbol parts of each $\boldsymbol{a}_{j}$ are independent of $j$. Then there exists a symbol $\boldsymbol{a}(y, \eta) \in \mathcal{R}^{\nu}\left(\Omega \times \mathbb{R}^{q} ;(\gamma, \gamma-\mu),\left(k, l ; j_{-}, j_{+}\right)\right)_{S, T}$ such that, for all $N \in \mathbb{N}$,

$$
\boldsymbol{a}(y, \eta)-\sum_{j=0}^{N-1} \boldsymbol{a}_{j}(y, \eta) \in \mathcal{R}^{\nu-N}\left(\Omega \times \mathbb{R}^{q} ;(\gamma, \gamma-\mu),\left(k, l ; j_{-}, j_{+}\right)\right)_{S, T} .
$$

Proposition 5.21. The pointwise composition of $\boldsymbol{a}(y, \eta) \in \mathcal{R}^{\nu}\left(\Omega \times \mathbb{R}^{q} ;(\gamma, \gamma-\mu),\left(k, \widetilde{l}_{j} j_{-}, \widetilde{j}_{+}\right)\right)_{S, R}$ and $\widetilde{\boldsymbol{a}}(y, \eta) \in \mathcal{R}^{\widetilde{\nu}}\left(\Omega \times \mathbb{R}^{q} ;(\gamma-\mu, \gamma-\mu-\widetilde{\mu}),\left(\widetilde{l}, l ; \widetilde{j}_{+}, j_{+}\right)\right)_{R, T}$ yields a symbol

$$
\widetilde{\boldsymbol{a}}(y, \eta) \boldsymbol{a}(y, \eta) \in \mathcal{R}^{\nu+\widetilde{\nu}}\left(\Omega \times \mathbb{R}^{q} ;(\gamma, \gamma-\mu-\widetilde{\mu}),\left(k, l ; j_{-}, j_{+}\right)\right)_{S, T}
$$

THEOREM 5.22. Let the notation be as in the previous Proposition 5.21 with $\Omega=\mathbb{R}^{q}$ and both $\widetilde{\boldsymbol{a}}(y, \eta)$ and $\boldsymbol{a}(y, \eta)$ be independent of $y$ for large $|y|$. Then there exists a symbol

$$
(\widetilde{\boldsymbol{a}} \# \boldsymbol{a})(y, \eta) \in \mathcal{R}^{\nu+\widetilde{\nu}}\left(\Omega \times \mathbb{R}^{q} ;(\gamma, \gamma-\mu-\widetilde{\mu}),\left(k, l ; j_{-}, j_{+}\right)\right)_{S, T},
$$

the so-called Leibniz-product of $\widetilde{\boldsymbol{a}}$ and $\boldsymbol{a}$, such that $\operatorname{Op}(\widetilde{\boldsymbol{a}}) \operatorname{Op}(\boldsymbol{a})=\operatorname{Op}(\widetilde{\boldsymbol{a}} \# \boldsymbol{a})$. If $\nu=\mu$ and $\widetilde{\nu}=\widetilde{\mu}$ then

$$
\sigma_{\wedge}(\widetilde{\boldsymbol{a}} \# \boldsymbol{a})(y, \eta)=\sigma_{\wedge}(\widetilde{\boldsymbol{a}})(y, \eta) \sigma_{\wedge}(\boldsymbol{a})(y, \eta), \quad \sigma_{M}(\widetilde{\boldsymbol{a}} \# \boldsymbol{a})(y, z)=\left(T^{\mu} \sigma_{M}(\widetilde{\boldsymbol{a}})\right)(y, z) \sigma_{M}(\boldsymbol{a})(y, z),
$$

where $T^{\sigma}$ acts on functions by shifting the argument, $\left(T^{\sigma} f\right)(z)=f(z+\sigma)$.

The proofs of Propositions 5.19, 5.21 and Theorems 5.20, 5.22 are somewhat lengthy and laborious, nevertheless elementary in the sense that they only rely on the correponding properties of standard edge symbols and general operator-valued symbols (and (5.17) for Proposition 5.19). Therefore we omit these proofs. 


\section{Operators of non-trivial type on manifolds with edges}

Let us begin this section by introducing a useful notation that we shall use frequently throughout the sequel: If $u, v$ are real-valued, continuous functions on a topological space, we shall write $u \prec v$ if $v \equiv 1$ on an open neighborhood containing the support of $u$.

6.1. Manifolds with edges and Sobolev spaces. The analysis of our classes of edgepseudodifferential operators takes place on (the interior of) a smooth manifold with boundary $\mathbb{M}$, that near the boundary has the structure of a fibre bundle over a smooth closed base space and where the fibre is a cone over a smooth closed manifold (both base space and cross section of the cone without boundary). For simplicity, we shall assume in this paper that this bundle is trivial. In case of trivial cross section (i.e., a point) we obtain a usual manifold with smooth boundary.

More precisely, there exists a homeomorphism of a neighborhood $V$ of $\partial \mathbb{M}$ to $Y \times[0,1) \times X$, where $X$ and $Y$ are smooth closed manifolds, that restricts to a diffeomorphism between $V \backslash \partial \mathbb{M}$ and $Y \times(0,1) \times X$. This gives rise to a splitting of coordinates that we shall keep fixed from now on. By identifying points $(y, 0, x)$ and $\left(y, 0, x^{\prime}\right)$ for any $x, x^{\prime} \in X$, we may obtain from $\mathbb{M}$ a topological space $M$ which is regarded as a manifold with edge $Y$ and model-cone $X^{\wedge}$. In order to emphasise this geometric structure, we shall often write $M$ instead of $\mathbb{M}$.

We shall now define weighted edge Sobolev spaces with and without asymptotics. To this end we assume that the asymptotic type $S$ is as in (5.1) but, additionally satisfies the so-called shadow condition: If $(p, m, L) \in S$ and $\operatorname{Re} p-1 \geq \frac{n+1}{2}-\gamma$ then there exists $(\widetilde{p}, \widetilde{m}, \widetilde{L}) \in S$ with $\widetilde{p}=p-1$, $m \leq \widetilde{m}$ and $L \subset \widetilde{L}$. This property ensures, in particular, that the operator of multiplication by any function $\varphi \in \mathcal{S}\left(\overline{\mathbb{R}}_{+}\right)$maps $\mathcal{K}^{s, \gamma}\left(X^{\wedge}\right)_{S}$ into itself.

Let us fix a covering of $Y$ by coordinate systems $\chi_{j}: U_{j} \subset Y \rightarrow \Omega_{j} \subset \mathbb{R}^{q}, j=1, \ldots, N$, and a system of functions $\varphi_{j}, \psi_{j} \in C_{0}^{\infty}\left(U_{j}\right)$ such that $\varphi_{1}, \ldots, \varphi_{N}$ form a partition of unity, and $\varphi_{j} \prec \psi_{j}$ for each $1 \leq j \leq N$. Moreover, $\omega \in C_{0}^{\infty}([0,1[)$ is a cut-off function, and we consider $(1-\omega)$ as a function on $\mathbb{M}$ that vanishes near the boundary as well as a function on the double $2 \mathbb{M}$ (by extension by 0 ).

Definition 6.1. The space $\mathcal{W}^{s, \gamma ; g}(M)$ for $g, s, \gamma \in \mathbb{R}$ consists of all distributions $u$ on $\mathbb{M} \backslash \partial \mathbb{M}$ such that $(1-\omega) u \in H^{s}(2 \mathbb{M})$ and

$$
\chi_{j *}\left(\varphi_{j} \omega u\right) \in \mathcal{W}^{s}\left(\mathbb{R}^{q}, \mathcal{K}^{s, \gamma ; g}\left(X^{\wedge}\right)\right) \quad \text { for all } 1 \leq j \leq N
$$

(cf. Remark 4.6), where $\chi_{j *}$ denotes the push-forward of distributions from $U_{j} \times(0,1) \times X$ to $\Omega_{j} \times(0,1) \times X$ under the map $(y, r, x) \mapsto\left(\chi_{j}(y), r, x\right)$.

In an analogous manner we define $\mathcal{W}^{s, \gamma ; g}(M)_{S}$ and subspaces $\mathcal{W}_{\varepsilon}^{s, \gamma ; g}(M)_{(S)}$ for some $\varepsilon>0$.

Up to equivalence of norms, the previous definitions are independent of the involved data (cutoff function, partition of unity, etc.), see Theorems 4 and 19 in Section 3.2.5 of [11]. Clearly Definition 6.1 has a straightforward extension to sections of vector-bundles $E$ over $M$, yielding a scale $\mathcal{W}^{s, \gamma ; g}(M, E)$. For simplicity of the presentation, however, we shall restrict ourselves to trivial bundles, i.e.,

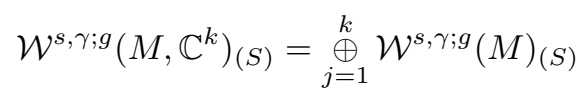

and analogously subspaces with asymptotics $Q \in \operatorname{As}(\gamma, \theta)$. 
In the following, we again restrict ourselves to the case $g=0$, having in mind that the (analogous) results remain valid also for arbitrary $g$, in particular, for $g=s-\gamma$.

6.2. Global projections in edge Sobolev spaces with asymptotics. In the construction of our new symbol algebra we made often use of the canonical splitting

$$
\mathcal{W}^{s}\left(\mathbb{R}^{q}, \mathcal{K}^{s, \gamma}\left(X^{\wedge}, \mathbb{C}^{k}\right)_{S}\right)=\mathcal{W}^{s}\left(\mathbb{R}^{q}, \mathcal{K}^{s, \gamma}\left(X^{\wedge}, \mathbb{C}^{k}\right)\right) \oplus \mathcal{V}^{s}\left(\mathbb{R}^{q}, \mathcal{E}_{S}\left(X^{\wedge}, \mathbb{C}^{k}\right)\right)
$$

and the corresponding projection $\mathrm{Op}\left(p_{0, S}\right)$. Passing to the manifold with edges $W$, we canonically can speak of $\mathcal{W}^{s, \gamma}\left(M, \mathbb{C}^{k}\right)_{S}$ and its subspace $\mathcal{W}^{s, \gamma}\left(M, \mathbb{C}^{k}\right)$, but there is not such a canonical choice of a complementing space. To overcome this problem, we shall prove in this subsection the following Theorem 6.2.

THEOREM 6.2. There exists an operator $P_{0, S}=P_{0, S}(k)$ having the following properties, simultaneously for all $s \in \mathbb{R}$ :

a) $P_{0, S} \in \mathcal{L}\left(\mathcal{W}^{s, \gamma}\left(M, \mathbb{C}^{k}\right)_{S}\right)$ is a projection onto $\mathcal{W}^{s, \gamma}\left(M, \mathbb{C}^{k}\right)$.

Moreover, $P_{0, S}$ is a pseudodifferential operator in the following sense:

b) If $\varphi, \psi \in C^{\infty}(\mathbb{M})$ are located in a chart $U \times[0,1) \times X$ with coordinate map $\chi: U \rightarrow \Omega \subset \mathbb{R}^{q}$, then

$$
\varphi P_{0, S} \psi=\varphi \chi_{*} \mathrm{Op}\left(p_{0, S}+r\right) \psi,
$$

where $p_{0, S}(\eta)$ is as in (5.3) (with $[\eta]$ now referring to the local expression for a smoothed norm function on the cotangent bundle of the edge $Y$ ),

$$
r(y, \eta) \in S_{\mathrm{cl}}^{-1}\left(\Omega \times \mathbb{R}^{q} ; \mathcal{K}^{s, \gamma}\left(X^{\wedge}, \mathbb{C}^{k}\right)_{S}, \mathcal{S}^{\infty}\left(X^{\wedge}, \mathbb{C}^{k}\right)\right),
$$

and $r(y, \eta) \equiv 0$ on $\mathcal{K}^{s, \gamma}\left(X^{\wedge}, \mathbb{C}^{k}\right)$.

c) If $\omega_{1}, \omega_{2} \in C^{\infty}\left(\left[0,1[)\right.\right.$ are cut-off functions with $\omega_{2} \prec \omega_{1}$ then

$$
\left(1-\omega_{1}\right) P_{0, S} \omega_{2}, \omega_{2} P_{0, S}\left(1-\omega_{1}\right): \mathcal{W}^{s, \gamma}\left(M, \mathbb{C}^{k}\right)_{S} \longrightarrow \mathcal{W}^{\infty, \infty}\left(M, \mathbb{C}^{k}\right),
$$

both vanishing on $\mathcal{W}^{s, \gamma}\left(M, \mathbb{C}^{k}\right)$.

d) If $\omega_{1}, \omega_{2} \in C^{\infty}\left(\left[0,1[)\right.\right.$ are cut-off functions and $\varphi_{1}, \varphi_{2} \in C^{\infty}(Y)$ have disjoint support, then

$$
\left(\omega_{1} \varphi_{1}\right) P_{0, S}\left(\omega_{2} \varphi_{2}\right): \mathcal{W}^{s, \gamma}\left(M, \mathbb{C}^{k}\right)_{S} \longrightarrow \mathcal{W}^{\infty, \infty}\left(M, \mathbb{C}^{k}\right)
$$

vanishing on $\mathcal{W}^{s, \gamma}\left(M, \mathbb{C}^{k}\right)$.

In $\mathrm{b}), \chi_{*}$ denotes the operator pull-back under the map $(y, r, x) \mapsto(\chi(y), r, x)$.

By virtue of property a), we have that $P_{0, S} \varphi=\varphi$ whenever $\varphi \in C^{\infty}(\mathbb{M})$ vanishes to infinite order at the boundary (respectively to sufficiently high order, depending on the asymptotic type $S$ ).

Property b) of the previous theorem says that locally, near the edge, $P_{0, S}$ coincides with $\operatorname{Op}\left(p_{0, S}\right)$, cf. Proposition 5.11, modulo a singular Green operator of order -1 , that additionally generates infinite flatness. In particular, the local edge symbols $\sigma_{\wedge}\left(p_{0, S}\right)(y, \eta)$ globally lead to a vector-bundle homomorphism

$$
\sigma_{\wedge}\left(P_{0, S}\right):\left(T^{*} Y \backslash 0\right) \times \mathcal{K}^{s, \gamma}\left(X^{\wedge}, \mathbb{C}^{k}\right)_{S} \longrightarrow\left(T^{*} Y \backslash 0\right) \times \mathcal{K}^{s, \gamma}\left(X^{\wedge}, \mathbb{C}^{k}\right)_{S} .
$$


Note that different choices of projections, say $P_{0, S}$ and $\widetilde{P}_{0, S}$, having the properties stated in the theorem give rise to the same principal edge symbol (6.2). Moreover,

$$
P_{0, S}-\widetilde{P}_{0, S}: \mathcal{W}^{s, \gamma}\left(M, \mathbb{C}^{k}\right)_{S} \longrightarrow \mathcal{W}^{s-1, \infty}\left(M, \mathbb{C}^{k}\right),
$$

vanishing on $\mathcal{W}^{s, \gamma}\left(M, \mathbb{C}^{k}\right)$.

Proof of Theorem 6.2. Without loss of generality we assume $k=1$.

Recall that we have identified a collar neighbourhood of $\partial \mathbb{M}$ in $\mathbb{M}$ with $Y \times[0,1[\times X$. Let us fix a covering $Y=\bigcup_{i=1}^{N} U_{i}$ with coordinate charts $\chi_{i}: U_{i} \rightarrow \Omega_{i} \subset \mathbb{R}^{q}$ and a subordinate partition of unity $\left\{\varphi_{i} \in C_{0}^{\infty}\left(U_{i}\right): i=1, \ldots, N\right\}$. Moreover choose $\psi_{i} \in C_{0}^{\infty}\left(U_{i}\right)$ with $\varphi_{i} \prec \psi_{i}$, and let $\omega, \widetilde{\omega} \in C_{0}^{\infty}([0,1[)$ be cut-off functions (viewed as functions on $\mathbb{M}$, supported near the boundary) with $\omega \prec \widetilde{\omega}$. Then we set

$$
P_{0, S}=\sum_{i=1}^{N}\left(\omega \varphi_{i}\right) \chi_{i *} \operatorname{Op}\left(p_{0, i}\right)\left(\psi_{i} \widetilde{\omega}\right)+(1-\omega), \quad p_{0, i}(y, \eta)=1-\kappa_{[\eta]} b_{S} \kappa_{[\eta]}^{-1},
$$

with $[\eta]$ denoting the expression in local coordinates $y \in \Omega_{i}$ of a smoothed norm function on the cotangent-bundle over $Y$, and $b_{S}$ being the projection in $\mathcal{K}^{s, \gamma}\left(X^{\wedge}\right)_{S}$ onto $\mathcal{K}^{s, \gamma}\left(X^{\wedge}\right)$ along $\mathcal{E}_{S}\left(X^{\wedge}\right)$, cf. (5.3).

By construction, it is clear that $P_{0, S}=1$ on $\mathcal{W}^{s, \gamma}(M)$ and that $P_{0, S}\left(\mathcal{W}^{s, \gamma}(M)_{S}\right)=\mathcal{W}^{s, \gamma}(M)$. Since $\mathcal{W}^{s, \gamma}(M)$ is a closed subspace of $\mathcal{W}^{s, \gamma}(M)_{S}$, we obtain a).

For b) we first have a look how an operator of the form $\mathrm{Op}\left(p_{0, i}\right)$ behaves under coordinate changes (in the edge variable). By the standard formula of expressing a pseudodifferential operator in new coordinates,

$$
\left.\left.\left(\chi_{*} p\right)(x, \xi)\right|_{x=\chi(y)} \sim \sum_{\alpha} \frac{1}{\alpha !}\left(D_{\eta}^{\alpha} p\right)\left(y,{ }^{t} \chi^{\prime}(y) \xi\right) D_{z}^{\alpha} e^{i \kappa(y, z) \xi}\right|_{z=y}
$$

with $\kappa(y, z)=\chi(z)-\chi(y)-\chi^{\prime}(y)(z-y)$, applied to $p_{0, i}$ and using (5.17), we obtain that $\mathrm{Op}\left(p_{0, i}\right)$ on $\left(\Omega_{i} \cap \Omega\right) \times X^{\wedge}$ under the coordinate change $\chi_{i} \circ \chi$ transforms to $\operatorname{Op}\left(p_{0, S}+r\right)$ with $p_{0, S}$ and $r$ as described in the statement. Thus, choosing a cut-off function $\omega_{0}$ with $\omega_{0} \prec \omega$ and using $P_{0, S}\left(1-\omega_{0}\right)=\left(1-\omega_{0}\right)$, we find

$$
\varphi P_{0, S} \psi=\varphi P_{0, S} \omega_{0} \psi+\varphi\left(1-\omega_{0}\right) \psi=\varphi \chi_{*} \mathrm{Op}\left(p_{0, S}+r\right) \omega_{0} \psi+\varphi\left(1-\omega_{0}\right) \psi .
$$

By the properties of $p_{0, S}$ and $r$ we find $\varphi \chi_{*} \operatorname{Op}\left(p_{0, S}+r\right)\left(\omega_{0}-1\right) \psi=-\varphi\left(1-\omega_{0}\right) \psi$ and this obviously yields $\varphi P_{0, S} \psi=\varphi \chi_{*} \mathrm{Op}\left(p_{0, S}+r\right) \psi$.

c), d) First of all, observe that all operators in question vanish on $\mathcal{W}^{s, \gamma}(M)$, since on that space $P_{0, S}$ coincides with the identity operator. For the same reason, we even have $\omega_{2} P_{0, S}\left(1-\omega_{1}\right)=0$ on $\mathcal{W}^{s, \gamma}(M)_{S}$.

By definition of $P_{0, S}$ in (6.3), the statement will follow if we verify corresponding properties for the local operators $R_{1}:=\left(1-\omega_{1}\right) \mathrm{Op}\left(p_{0, i}\right) \omega_{2}$ and $R_{2}:=\widetilde{\varphi}_{1} O p\left(p_{0, i}\right) \widetilde{\varphi}_{2}$, where $\widetilde{\varphi}_{1}, \widetilde{\varphi}_{2} \in C^{\infty}\left(\Omega_{i}\right)$ have disjoint support. To show this let, without loss of generality, $\Omega_{i}=\mathbb{R}^{q}$ and $[\cdot]$ be independent of $y$ for large $|y|$. By using the explicit representation (5.16), and writing $\left(1-\omega_{1}\right)=t^{N}\left(t^{-N}\left(1-\omega_{1}\right)\right)$ for arbitrary $N \in \mathbb{N}$, it is straightforward to see that $R_{1}=\mathrm{Op}\left(r_{1}\right)$ with a symbol $r_{1} \in S^{-N}\left(\mathbb{R}^{q} \times\right.$ $\left.\mathbb{R}^{q} ; \mathcal{K}^{s, \gamma}\left(X^{\wedge}\right)_{S}, \mathcal{S}^{\infty}\left(X^{\wedge}\right)\right)$. This yields $R_{1}: \mathcal{W}^{s, \gamma}\left(\mathbb{R}^{q} \times X^{\wedge}\right)_{S} \rightarrow \mathcal{W}^{\infty, \infty}\left(\mathbb{R}^{q} \times X^{\wedge}\right)$. The same is true for $R_{2}$, using integration by parts and (5.17). 
REMARK 6.3. Adapting methods and results of [2] that show that, for a smooth compact manifold $\Omega$ with boundary, $H^{s}(\Omega) / H_{0}^{s}(\Omega)$ is isomorphic to $H^{s}\left(\partial \Omega, \mathbb{C}^{\left[s-\frac{1}{2}\right]}\right)$, where $H_{0}^{s}(\Omega)$ denotes the closure of $C_{0}^{\infty}($ int $\Omega)$ in $H^{s}(\Omega)$, one can show that

$$
\operatorname{ker} P_{0, S} \cong \mathcal{W}^{s, \gamma}\left(M, \mathbb{C}^{k}\right)_{S} / \mathcal{W}^{s, \gamma}\left(M, \mathbb{C}^{k}\right) \cong H^{s}\left(Y, \mathbb{C}^{k} \otimes \mathbb{C}^{\iota(S)}\right),
$$

where $\iota(S)$ is the dimension of $\mathcal{E}_{S}\left(X^{\wedge}\right)$, cf. (4.3).

6.3. Global regular and singular Green operators. The full algebra of operators on a manifold with edges will consist, roughly speaking, of pseudodifferential operators that locally near the edge can be represented as an operator with symbol from the edge symbol algebra introduced in Definition 5.16. There are also non-local operators in this algebra which we shall introduce first.

Definition 6.4. A smoothing operator (associated with asymptotic types $S, T$, vector bundles $\mathbb{C}^{k}$, $\mathbb{C}^{l}, J_{-}, J_{+}$, and with weight-data $\left.(\gamma, \gamma-\mu)\right)$ is an operator $\mathcal{C}$ such that, for all $s \in \mathbb{R}$,

$$
\mathcal{C}=\mathcal{C}_{0}\left(\begin{array}{cc}
P_{0, S} & 0 \\
0 & 1
\end{array}\right)+\left(\begin{array}{cc}
C_{11} & 0 \\
C_{21} & 0
\end{array}\right): \begin{array}{ccc}
\mathcal{W}^{s, \gamma}\left(M, \mathbb{C}^{k}\right)_{S} & \mathcal{W}^{\infty, \gamma-\mu}\left(M, \mathbb{C}^{l}\right)_{T} \\
H^{s}\left(Y, J_{-}\right) & \oplus & \oplus \\
& & H^{\infty}\left(Y, J_{+}\right)
\end{array}
$$

where

a) $P_{0, S}$ is a projection as in Theorem 6.2 ,

b) both $C_{11}$ and $C_{21}$ vanish on $\mathcal{W}^{s, \gamma}\left(M, \mathbb{C}^{k}\right)$, and there exists an $\varepsilon=\varepsilon\left(C_{11}\right)>0$ such that $C_{11}: \mathcal{W}^{s, \gamma}\left(M, \mathbb{C}^{k}\right) \rightarrow \mathcal{W}_{\varepsilon}^{\infty, \gamma-\mu}\left(M, \mathbb{C}^{l}\right)_{T}$

c) for any $r \in \mathbb{R}$

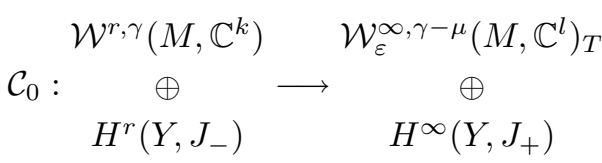

for some $\varepsilon=\varepsilon\left(\mathcal{C}_{0}\right)>0$, while its adjoint $\mathcal{C}_{0}^{*}$ induces maps

$$
\begin{aligned}
& \mathcal{W}^{r,-\gamma+\mu+\tau}\left(M, \mathbb{C}^{l}\right) \quad \mathcal{W}_{\varepsilon}^{\infty,-\gamma}\left(M, \mathbb{C}^{k}\right) \\
& \begin{array}{cccc}
\mathcal{C}_{0}^{*}: & \oplus & \longrightarrow & \oplus \\
& H^{r}\left(Y, J_{+}\right) & & H^{\infty}\left(Y, J_{-}\right)
\end{array} .
\end{aligned}
$$

The space of all such operators we shall denote by

$$
\mathcal{Y}^{-\infty}\left(M ;(\gamma, \gamma-\mu),\left(k, l ; J_{-}, J_{+}\right)\right)_{S, T}
$$

If in (6.4) we can choose $C_{11}=0$ and $C_{21}=0$, we call $\mathcal{C}$ a regular smoothing operator. If $\mathcal{C}_{0}$ is represented as a block matrix, $\mathcal{C}_{0}=\left(\begin{array}{ll}C_{11}^{0} & C_{12}^{0} \\ C_{21}^{0} & C_{22}^{0}\end{array}\right)$, and we can choose in (6.4) both $C_{11}^{0}=0$ and $C_{21}^{0}=0$, we call $\mathcal{C}$ a singular smoothing operator.

Let us remark that - after fixing the choice of $P_{0, S}$ - the decomposition (6.4) uniquely determines $\mathcal{C}_{0}, C_{11}$, and $C_{21}$.

For the definition of global Green operators let $Y=\bigcup_{i=1}^{N} U_{i}$ with coordinate charts $\chi_{i}: U_{i} \rightarrow$ $\Omega_{i} \subset \mathbb{R}^{q}$ over which $J_{ \pm}$is trivial with trivializations $\chi_{i, \pm}:\left.J_{ \pm}\right|_{U_{i}} \longrightarrow \Omega_{i} \times \mathbb{C}^{j_{ \pm}}$. Let $\varphi_{1}, \ldots, \varphi_{N}$ be a corresponding partition of unity on $Y$ and $\psi_{i} \in C_{0}^{\infty}\left(U_{i}\right)$ such that $\varphi_{i} \prec \psi_{i}$. Moreover, let $\omega, \widetilde{\omega} \in C_{0}^{\infty}([0,1))$ be cut-off functions with $\omega \prec \widetilde{\omega}$. 
Definition 6.5. A Green operator of order $\nu \in \mathbb{R}$ (associated with asymptotic types $S, T$, vector bundles $\mathbb{C}^{k}, \mathbb{C}^{l}, J_{-}, J_{+}$, and with weight-data $\left.(\gamma, \gamma-\mu)\right)$ is any operator $\mathcal{G}$ of the form

$$
\mathcal{G}=\sum_{i=1}^{N}\left(\begin{array}{cc}
\omega \varphi_{i} \circ \chi_{i}^{*} & 0 \\
0 & \varphi_{i} \circ \chi_{i,+}^{*}
\end{array}\right) \operatorname{Op}\left(g_{i}\right)\left(\begin{array}{cc}
\left(\chi_{i}\right)_{*} \circ \omega \psi_{i} & 0 \\
0 & \left(\chi_{i,-}\right)_{*} \circ \psi_{i}
\end{array}\right)+\mathcal{C}
$$

( $\chi^{*}$ and $\chi_{*}$ denote the pull-back and push-forward, respectively, of sections under $\chi$ ), where $\mathcal{C}$ is a smoothing operator in the sense of Definition 6.4 and each $g_{i}$ is a $\nu$-th order Green symbol as in Definition 5.15, $g_{i} \in \mathcal{R}_{G}^{\nu}\left(\Omega_{i} \times \mathbb{R}^{q} ;(\gamma, \gamma-\mu),\left(k, l ; j_{-}, j_{+}\right)\right)_{S, T}$. The space of all such operators is denoted by

$$
\mathcal{Y}_{G}^{\nu}\left(M ;(\gamma, \gamma-\mu),\left(k, l ; J_{-}, J_{+}\right)\right)_{S, T}
$$

\subsection{The algebra of edge operators on $W$.}

Definition 6.6. Let $\mu, \nu \in \mathbb{R}$ with $\mu-\nu \in \mathbb{N}$. The space

$$
\mathcal{Y}^{\nu}\left(M ;(\gamma, \gamma-\mu),\left(k, l ; J_{-}, J_{+}\right)\right)_{S, T}
$$

consists of all operators

$$
\mathcal{A}=\left(\begin{array}{cc}
A P_{0, S} & 0 \\
0 & 0
\end{array}\right)+\mathcal{G}: \begin{array}{ccc}
\mathcal{W}^{s, \gamma}\left(M, \mathbb{C}^{k}\right)_{S} & \oplus \\
H^{s}\left(Y, J_{-}\right) & \longrightarrow & \mathcal{W}^{s-\mu, \gamma-\mu}\left(M, \mathbb{C}^{l}\right)_{T} \\
& \longrightarrow & H^{s-\mu}\left(Y, J_{+}\right)
\end{array}
$$

where $P_{0, S}$ is as in Theorem $6.2, \mathcal{G} \in \mathcal{Y}_{G}^{\nu}\left(M ;(\gamma, \gamma-\mu),\left(k, l ; J_{-}, J_{+}\right)\right)_{S, T}$, and $A$ is an operator from the standard edge algebra $Y^{\nu}(M ;(\gamma, \gamma-\mu),(k, l))$, cf. Section 8 .

REMARK 6.7. Throughout the paper we admit also the case $\sigma=\tau=0$, i.e., that the asymptotic types $S$ and $T$ disappear, cf. Remark4.9. In that case we recover the standard edge algebra $\mathcal{Y}^{\nu}(M ;(\gamma, \gamma-$ $\left.\mu),\left(k, l ; J_{-}, J_{+}\right)\right)$.

Similarly as in (6.5), each operator $\mathcal{A} \in \mathcal{Y}^{\nu}\left(M ;(\gamma, \gamma-\mu),\left(k, l ; J_{-}, J_{+}\right)\right)_{S, T}$ can be expressed locally near the edge in terms of edge symbols belonging to $\mathcal{R}^{\nu}\left(\Omega_{i} \times \mathbb{R}^{q} ;(\gamma, \gamma-\mu),\left(k, l ; j_{-}, j_{+}\right)\right)_{S, T}$.

Definition 6.8. Let $\mathcal{A} \in \mathcal{Y}^{\mu}\left(M ;(\gamma, \gamma-\mu),\left(k, l ; J_{-}, J_{+}\right)\right)_{S, T}$ be as in $(6.6)$ with $\nu=\mu$. Let us denote by $\pi_{Y}: T^{*} Y \backslash 0 \rightarrow Y$ the canonical projection. Then the principal edge symbol of $\mathcal{A}$ is the vector-bundle homomorphism

$$
\begin{aligned}
\sigma_{\wedge}(\mathcal{A}) & =\left(\begin{array}{cc}
\sigma_{\wedge}(A) \sigma_{\wedge}\left(P_{0, S}\right) & 0 \\
0 & 0
\end{array}\right)+\sigma_{\wedge}(\mathcal{G}): \\
& \pi_{Y}^{*}\left(\begin{array}{c}
\mathcal{K}^{s, \gamma}\left(X^{\wedge}, \mathbb{C}^{k}\right)_{S} \\
\oplus \\
J_{-}
\end{array}\right) \longrightarrow \pi_{Y}^{*}\left(\begin{array}{c}
\mathcal{K}^{s-\mu, \gamma-\mu}\left(X^{\wedge}, \mathbb{C}^{l}\right)_{T} \\
\oplus \\
J_{+}
\end{array}\right), \quad s \in \mathbb{R},
\end{aligned}
$$

where $\sigma_{\wedge}(A)$ is the principal edge symbol of standard edge operators, cf. Section 8 , and $\sigma_{\wedge}\left(P_{0, S}\right)$ is as in (6.2). Moreover, the conormal symbol of $\mathcal{A}$ is, by definition,

$$
\sigma_{M}(\mathcal{A})(y, z)=\sigma_{M}(A)(y, z): H^{s}(X) \longrightarrow H^{s-\mu}(X), \quad s \in \mathbb{R},
$$

where $y \in Y$ and $z \in \mathbb{C}$ with $\operatorname{Re} z=\frac{n+1}{2}-\gamma$, cf. Section 8 . 
Occcasionally, we prefer to write the action of the principal edge symbol in each fibre, i.e.,

$$
\sigma_{\wedge}(\mathcal{A})(y, \eta): \begin{gathered}
\mathcal{K}^{s, \gamma}\left(X^{\wedge}, \mathbb{C}^{k}\right)_{S} \\
\oplus \\
J_{-, y}
\end{gathered}
$$

Besides principal edge and conormal symbol we have the standard interior pseudodifferential symbol

$$
\sigma_{\psi}(\mathcal{A}):=\sigma_{\psi}(A) \in C^{\infty}\left(T^{*}(M \backslash Y) \backslash 0\right),
$$

cf. (8.8), and the rescaled symbol

$$
\widetilde{\sigma}_{\psi}(\mathcal{A}):=\widetilde{\sigma}_{\psi}(A) \in C^{\infty}\left(\left(T^{*} \partial \mathbb{M} \times \mathbb{R}\right) \backslash 0\right),
$$

cf. (8.9). Based on the algebra property of the standard edge algebra and the composition result Theorem 5.22, one can show the following:

TheOREM 6.9. Let $\mathcal{A} \in \mathcal{Y}^{\nu}\left(M ;(\gamma, \gamma-\mu),\left(k, \widetilde{l} ; J_{-}, \widetilde{J}_{+}\right)\right)_{S, R}$ and $\widetilde{\mathcal{A}} \in \mathcal{Y}^{\widetilde{\nu}}(M ;(\gamma-\mu, \gamma-\mu-$ $\left.\widetilde{\mu}),\left(\widetilde{l}, l ; \widetilde{J}_{+}, J_{+}\right)\right)_{R, T}$. Then

$$
\widetilde{\mathcal{A}} \mathcal{A} \in \mathcal{Y}^{\nu+\widetilde{\nu}}\left(M ;(\gamma, \gamma-\mu-\widetilde{\mu}),\left(k, l ; J_{-}, J_{+}\right)\right)_{S, T} .
$$

Moreover, in case $\nu=\mu$ and $\widetilde{\nu}=\widetilde{\mu}$,

$$
\sigma_{\wedge}(\widetilde{\mathcal{A}} \mathcal{A})(y, \eta)=\sigma_{\wedge}(\widetilde{\mathcal{A}})(y, \eta) \sigma_{\wedge}(\mathcal{A})(y, \eta), \quad \sigma_{M}(\widetilde{\mathcal{A}} \mathcal{A})(y, z)=\left(T^{\mu} \sigma_{M}(\widetilde{\mathcal{A}})\right)(y, z) \sigma_{M}(\mathcal{A})(y, z),
$$

where $T^{\sigma}$ is the shift operator, $\left(T^{\sigma} f\right)(z)=f(z+\sigma)$.

Similarly, using Theorem 5.20, we have:

Theorem 6.10. For $j \in \mathbb{N}$ let $\mathcal{A}_{j} \in \mathcal{Y}^{\mu-j}\left(M ;(\gamma, \gamma-\mu-\widetilde{\mu}),\left(k, l ; J_{-}, J_{+}\right)\right)_{S, T}$ such that the $\varepsilon$ weights involved in the Green parts of each $\mathcal{A}_{j}$ do not dependent on $j$. Then there exists an operator $\mathcal{A} \in \mathcal{Y}^{\mu}\left(M ;(\gamma, \gamma-\mu-\widetilde{\mu}),\left(k, l ; J_{-}, J_{+}\right)\right)_{S, T}$ such that, for all $N \in \mathbb{N}$,

$$
\mathcal{A}-\sum_{j=0}^{N-1} \mathcal{A}_{j} \in \mathcal{Y}^{\mu-N}\left(M ;(\gamma, \gamma-\mu-\widetilde{\mu}),\left(k, l ; J_{-}, J_{+}\right)\right)_{S, T} .
$$

\section{Ellipticity and parametrix}

In this section we introduce the notion of ellipticity in the edge calculus with singular trace and Green operators and show that elliptic operators possess a parametrix within the calculus. As a preliminary step, we consider in Section 7.1 the subalgebra of operators which coincide with the identity modulo Green operators. The general case is then treated in the subsequent Section 7.2.

Definition 7.1. An operator $\mathcal{A} \in \mathcal{Y}^{\mu}\left(M,(\gamma, \gamma-\mu),\left(k, k ; J_{-}, J_{+}\right)\right)_{S, T}$ is called elliptic if

(i) $\sigma_{\psi}(\mathcal{A})$ and $\widetilde{\sigma}_{\psi}(\mathcal{A})$ are pointwise invertible on $T^{*}(M \backslash Y) \backslash 0$ and $\left(T^{*} \partial \mathbb{M} \times \mathbb{R}\right) \backslash 0$, respectively,

(ii) $\sigma_{\wedge}(\mathcal{A})(y, \eta)$ defines isomorphisms (6.8) for all $(y, \eta) \in T^{*} Y \backslash 0$ and some $s \in \mathbb{R}$.

Note that if $\mathcal{A}=\left(\begin{array}{ll}A_{11} & A_{12} \\ A_{21} & A_{22}\end{array}\right)$ is elliptic, then

$$
\sigma_{\wedge}\left(A_{11}\right)(y, \eta): \mathcal{K}^{s, \gamma}\left(X^{\wedge}, \mathbb{C}^{k}\right)_{S} \longrightarrow \mathcal{K}^{s-\mu, \gamma-\mu}\left(X^{\wedge}, \mathbb{C}^{k}\right)_{T}
$$


is a family of Fredholm operators of index $j_{+}-j_{-}$, where $j_{ \pm}$denotes the fibre dimensions of $J_{ \pm}$. Under the ellipticity assumption (i), the Fredholm property of (7.1) in turn is equivalent to the bijectivity of the subordinate conormal symbol

$$
\sigma_{M}(\mathcal{A})(y, z): H^{s}(X) \rightarrow H^{s-\mu}(X), \quad s \in \mathbb{R},
$$

for all $y \in Y$ and $z \in \Gamma_{\frac{n+1}{2}-\gamma}$. In particular, it does not depend on the choice of $S$ and $T$, and the conormal symbol might have non-bijectivity points on the weight line $\Gamma_{\frac{n+1}{2}-(\gamma-\sigma)}$ (with $\sigma$ corresponding to the type $S$ ).

7.1. The inverse of identity plus Green symbol. For the following statement we use the notation $\mathcal{I}=\operatorname{diag}(1,1)$.

Proposition 7.2. Let $\mathcal{G} \in \mathcal{Y}_{G}^{0}(M,(\gamma, \gamma),(k, k ; J, J))_{S, S}$ such that $\sigma_{\wedge}(\mathcal{I}+\mathcal{G})(y, \eta)=\mathcal{I}+\sigma_{\wedge}(\mathcal{G})(y, \eta)$ is an isomorphism for each $y$ and $\eta \neq 0$. Then there exists a $\mathcal{C} \in \mathcal{Y}_{G}^{0}(M,(\gamma, \gamma),(k, k ; J, J))_{S, S}$ such that

$$
\left(\mathcal{I}+\sigma_{\wedge}(\mathcal{G})(y, \eta)\right)^{-1}=\mathcal{I}+\sigma_{\wedge}(\mathcal{C})(y, \eta) \quad \text { for all }(y, \eta) \in T^{*} Y \backslash 0 .
$$

Proof. For notational simplicity let $k=1$. Without loss of generality, we may assume $\gamma=0$. Let us write

$$
\mathcal{I}+\sigma_{\wedge}(\mathcal{G})(y, \eta)=:\left(\begin{array}{cc}
1+G_{11} & G_{12} \\
G_{21} & G_{22}
\end{array}\right)(y, \eta): \begin{array}{ccc}
\mathcal{K}^{0,0}\left(X^{\wedge}\right)_{S} & \mathcal{K}^{0,0}\left(X^{\wedge}\right)_{S} \\
J & \longrightarrow & \oplus \\
& J
\end{array}
$$

and let us suppress $(y, \eta)$ from the notation for a while. Since the following considerations are local in $(y, \eta)$, we assume that $J=\mathbb{C}^{j}$ for some $j \in \mathbb{N}$.

Let $b_{S}$ be the projection in $\mathcal{K}^{0,0}\left(X^{\wedge}\right)_{S}$ onto $\mathcal{E}_{S}\left(X^{\wedge}\right)$ along $\mathcal{K}^{0,0}\left(X^{\wedge}\right)$, cf. (5.3) and (5.15). Then

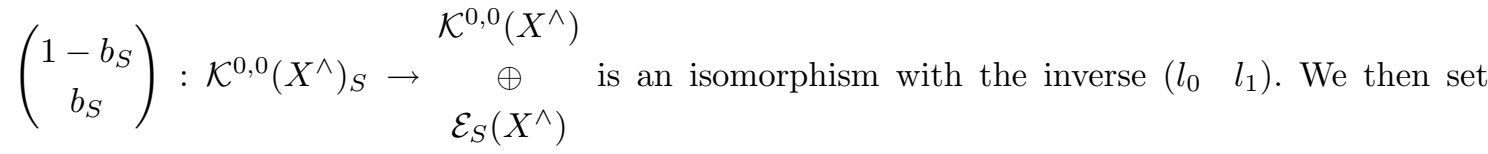
$\mathfrak{B}:=\left(\begin{array}{cc}1-b_{S} & 0 \\ b_{S} & 0 \\ 0 & 1\end{array}\right)$ and form the isomorphism $\mathfrak{B}\left(\mathcal{I}+\sigma_{\wedge}(\mathcal{G})\right) \mathfrak{B}^{-1}$ which equals

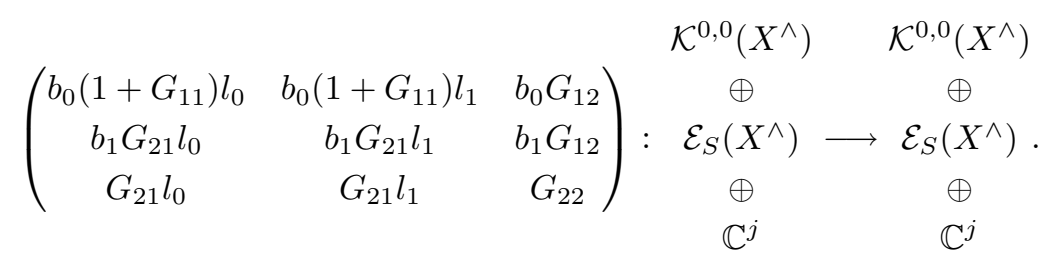

Using an isomorphism $\mathcal{E}_{S}\left(X^{\wedge}\right) \cong \mathbb{C}^{\iota(S)}$, cf. (4.3), and writing $G:=b_{0} G_{11} l_{0}$, we transform (7.3) to the form

$$
\left(\begin{array}{cc}
1+G & H_{12} \\
H_{21} & H_{22}
\end{array}\right): \underset{\mathbb{C}^{\iota(S)+j}}{\oplus} \longrightarrow \begin{gathered}
\mathcal{K}^{0,0}\left(X^{\wedge}\right) \\
\mathbb{C}^{\iota(S)+j}
\end{gathered}
$$


Now let $\left(y_{0}, \eta_{0}\right) \in S^{*} Y$ (the unit cosphere bundle) be fixed. Since isomorphisms form an open set and each matrix can be approximated arbitrarily well by an invertible one, there exists an invertible $(\iota(S)+j) \times(\iota(S)+j)$-matrix $\widetilde{H}_{22}$ such that

$$
\left(\begin{array}{cc}
1+G & H_{12} \\
H_{21} & \widetilde{H}_{22}
\end{array}\right): \begin{gathered}
\mathcal{K}^{0,0}\left(X^{\wedge}\right) \\
\mathbb{C}^{\iota(S)+j}
\end{gathered} \underset{\mathbb{C}^{\iota(S)+j}}{\bigoplus^{0,0}\left(X^{\wedge}\right)}
$$

is pointwise invertible in an open neighborhood of $\left(y_{0}, \eta_{0}\right)$ in $S^{*} Y$. From the decomposition

$$
\left(\begin{array}{cc}
1+G & H_{12} \\
H_{21} & \widetilde{H}_{22}
\end{array}\right)=\left(\begin{array}{cc}
1 & H_{12} \widetilde{H}_{22}^{-1} \\
0 & 1
\end{array}\right)\left(\begin{array}{cc}
(1+G)-H_{12} \widetilde{H}_{22}^{-1} H_{21} & 0 \\
0 & \tilde{H}_{22}
\end{array}\right)\left(\begin{array}{cc}
1 & 0 \\
\widetilde{H}_{22}^{-1} H_{21} & 1
\end{array}\right)
$$

we deduce that the second term on the right-hand side is invertible, since all the other blockmatrices are. In particular,

$$
1+D: \mathcal{K}^{0,0}\left(X^{\wedge}\right) \longrightarrow \mathcal{K}^{0,0}\left(X^{\wedge}\right), \quad D:=G-H_{12} \tilde{H}_{22}^{-1} H_{21},
$$

is invertible. But now $D$ is (the symbol of) a regular Green operator, i.e. a Green operator from the standard edge calculus. Then it is known that $(1+D)^{-1}=1+M$ with a (symbol of a) regular Green operator. This yields

$$
\left(\begin{array}{cc}
1+G & H_{12} \\
H_{21} & \widetilde{H}_{22}
\end{array}\right)^{-1}=\left(\begin{array}{cc}
1+M & -(1+M) H_{12} \widetilde{H}_{22}^{-1} \\
-\widetilde{H}_{22}^{-1} H_{21}(1+M) & \widetilde{H}_{22}^{-1}
\end{array}\right)=:\left(\begin{array}{cc}
1+M & N_{12} \\
N_{21} & N_{22}
\end{array}\right) .
$$

Then

$$
\left(\begin{array}{cc}
1+G & H_{12} \\
H_{21} & H_{22}
\end{array}\right)\left(\begin{array}{cc}
1+M & N_{12} \\
N_{21} & N_{22}
\end{array}\right)=\left(\begin{array}{cc}
1 & 0 \\
B & R
\end{array}\right)
$$

with an invertible matrix $R$, and it follows that

$$
\left(\begin{array}{cc}
1+G & H_{12} \\
H_{21} & H_{22}
\end{array}\right)^{-1}=\left(\begin{array}{cc}
1+M & N_{12} \\
N_{21} & N_{22}
\end{array}\right)\left(\begin{array}{cc}
1 & 0 \\
-R^{-1} B & R^{-1}
\end{array}\right)=:\left(\begin{array}{cc}
1+K & L_{12} \\
L_{21} & L_{22}
\end{array}\right),
$$

where the functions $K$ and $L_{i j}$ are smooth near $\left(y_{0}, \eta_{0}\right)$, and have the properties that

$$
K(y, \eta), K(y, \eta)^{*}: \mathcal{K}^{0,0}\left(X^{\wedge}\right) \longrightarrow \mathcal{S}_{\varepsilon}^{0}\left(X^{\wedge}\right), \quad L_{12}(y, \eta), L_{21}(y, \eta)^{*}: \mathbb{C}^{\iota(S)+j} \longrightarrow \mathcal{S}_{\varepsilon}^{0}\left(X^{\wedge}\right)
$$

for some $\varepsilon>0$.

Since the local inverses are unique, we thus obtain an inverse of the form (7.6) globally on $S^{*} Y$, having the mapping properties just described. It then remains to set

$$
\left(\begin{array}{cc}
1+C_{11} & C_{12} \\
C_{21} & C_{22}
\end{array}\right)=\mathfrak{B}^{-1}\left(\begin{array}{cc}
1+K & L_{12} \\
L_{21} & L_{22}
\end{array}\right) \mathfrak{B}
$$

and to define $\sigma_{\wedge}(\mathcal{C})(y, \eta)$ by

$$
\mathcal{I}+\sigma_{\wedge}(\mathcal{C})(y, \eta)=\operatorname{diag}\left(\kappa_{|\eta|}, 1\right)\left(\begin{array}{cc}
1+C_{11} & C_{12} \\
C_{21} & C_{22}
\end{array}\right)(y, \eta /|\eta|) \operatorname{diag}\left(\kappa_{|\eta|}^{-1}, 1\right) .
$$

By using a zero excision function $\chi(\eta)$, we then find a corresponding operator $\mathcal{C}$ having the constructed principal edge symbol. 


\subsection{The parametrix construction.}

Theorem 7.3. Let $\mathcal{A} \in \mathcal{Y}^{\mu}\left(M,(\gamma, \gamma-\mu),\left(k, k, J_{-}, J_{+}\right)\right)_{S, T}$ be elliptic. Then $\mathcal{A}$ has a parametrix $\mathcal{P} \in \mathcal{Y}^{-\mu}\left(M,(\gamma-\mu, \gamma),\left(k, k, J_{+}, J_{-}\right)\right)_{T, S}$, i.e.,

$$
\begin{aligned}
& \mathcal{A P}-\mathcal{I} \in \mathcal{Y}^{-\infty}\left(M,(\gamma-\mu, \gamma-\mu),\left(k, k, J_{+}, J_{+}\right)\right)_{T, T}, \\
& \mathcal{P} \mathcal{A}-\mathcal{I} \in \mathcal{Y}^{-\infty}\left(M,(\gamma, \gamma),\left(k, k, J_{-}, J_{-}\right)\right)_{S, S},
\end{aligned}
$$

where $\mathcal{I}=\operatorname{diag}(1,1)$ is the identity operator. In particular, $\mathcal{A}$ induces Fredholm operators

$$
\begin{aligned}
& \mathcal{W}^{s, \gamma}\left(M, \mathbb{C}^{k}\right)_{S} \quad \mathcal{W}^{s-\mu, \gamma-\mu}\left(M, \mathbb{C}^{k}\right)_{T} \\
& \mathcal{A}: \quad \oplus \quad \oplus \quad \oplus \quad, \quad s \in \mathbb{R} \text {. } \\
& H^{s}\left(Y, J_{-}\right) \quad H^{s-\mu}\left(Y, J_{+}\right)
\end{aligned}
$$

ProOF. Let us construct a left parametrix $\mathcal{P}$; the construction of a right-parametrix is similar. Then $\mathcal{P}$ is a two-sided parametrix, and we obtain the Fredholm property of $\mathcal{A}$, since the remainders are compact operators in the respective Sobolev spaces. For the construction of $\mathcal{P}$ we assume for simplicity that $k=1$ (the general case is completely analogous).

Writing $\mathcal{A}$ as a block-matrix $\mathcal{A}=\left(A_{i j}\right)_{i, j=1,2}$ with $A_{11}=A P_{0, S}$, cf. the formulas (6.6) and (7.1), condition (ii) of Definition 7.1 yields a family of Fredholm operators

$$
\sigma_{\wedge}(A)(y, \eta): \mathcal{K}^{s, \gamma}\left(X^{\wedge}\right) \longrightarrow \mathcal{K}^{s-\mu, \gamma-\mu}\left(X^{\wedge}\right), \quad(y, \eta) \in T^{*} Y \backslash 0,
$$

of index $l_{+}-l_{-}$for $l_{+}=j_{+}+\iota(T)$ and $l_{-}=j_{-}+\iota(S)$. Using the technique of constructing a parametrix in the standard edge calculus (cf. Theorem 8.5, below), we find an elliptic operator $\mathcal{B} \in \mathcal{Y}^{-\mu}\left(M,(\gamma-\mu, \gamma),\left(1,1 ; L_{+}, L_{-}\right)\right)$for $L_{+}=J_{+} \oplus \mathbb{C}^{\iota(T)}$ and $L_{-}=J_{-} \oplus \mathbb{C}^{\iota(S)}$, which has the following properties: The upper left corner $B$ of $\mathcal{B}$, viewed as an element of $L^{-\mu}(M \backslash Y)$, is a parametrix of the elliptic operator $A \in L^{\mu}(M \backslash Y)$, and $\left(T^{\mu} \sigma_{M}(B)\right) \sigma_{M}(A)=1$. Moreover, the operator $\mathcal{B}$ is Fredholm as a map

$$
\begin{array}{ccc}
\mathcal{W}^{s-\mu, \gamma-\mu}(M) & \mathcal{W}^{s, \gamma}(M) \\
\mathcal{B}: \begin{array}{c}
\oplus \\
H^{s-\mu}\left(Y, J_{+}\right) \oplus H^{s-\mu}\left(Y, \mathbb{C}^{\iota(T)}\right)
\end{array} & & H^{s}\left(Y, J_{-}\right) \oplus H^{s}\left(Y, \mathbb{C}^{\iota(S)}\right)
\end{array} .
$$

A simple algebraic argument, using the isomorphisms

$$
\mathcal{W}^{s, \gamma}(M) \oplus H^{s}\left(Y, \mathbb{C}^{\iota(S)}\right) \cong \mathcal{W}^{s, \gamma}(M)_{S}, \quad \mathcal{W}^{s-\mu, \gamma-\mu}(M) \oplus H^{s-\mu}\left(Y, \mathbb{C}^{\iota(T)}\right) \cong \mathcal{W}^{s-\mu, \gamma-\mu}(M)_{T},
$$

cf. Remark 6.3, allows us to reorganise the operator $\mathcal{B}$ to an operator

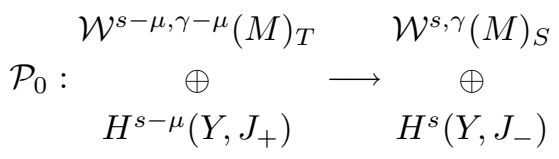

with $\mathcal{P}_{0} \in \mathcal{Y}^{-\mu}\left(M,(\gamma-\mu, \gamma),\left(1,1 ; J_{-}, J_{+}\right)\right)_{T, S}$ and such that

$$
\mathcal{G}:=\mathcal{P}_{0} \mathcal{A}-\mathcal{I} \in \mathcal{Y}_{G}^{0}\left(M,(\gamma, \gamma),\left(1,1 ; J_{-}, J_{-}\right)\right)_{S, S} .
$$

Both $\mathcal{P}_{0}$ and $\mathcal{A}$ are elliptic; thus also $\mathcal{I}+\mathcal{G}$ is elliptic. By Proposition 7.2 we find an operator $\mathcal{C} \in \mathcal{Y}_{G}^{0}\left(M,(\gamma, \gamma),\left(1,1 ; J_{-}, J_{-}\right)\right)_{S, S}$ such that

$$
(\mathcal{I}+\mathcal{C})(\mathcal{I}+\mathcal{G})=\mathcal{I}+\mathcal{K} \quad \text { for a } \quad \mathcal{K} \in \mathcal{Y}_{G}^{-1}\left(M,(\gamma, \gamma),\left(1,1 ; J_{-}, J_{-}\right)\right)_{S, S}
$$


Setting $\mathcal{P}_{1}=(\mathcal{I}+\mathcal{C}) \mathcal{P}_{0}$ we thus obtain $\mathcal{P}_{1} \mathcal{A}=\mathcal{I}+\mathcal{K}$. Applying Theorem 6.10, we can form the asymptotic sum

$$
\mathcal{L}:=\sum_{j=1}^{\infty}(-1)^{j} \mathcal{K}^{j} \in \mathcal{Y}_{G}^{-1}\left(M,(\gamma, \gamma),\left(1,1 ; J_{-}, J_{-}\right)\right)_{S, S}
$$

Then $\mathcal{P}:=(\mathcal{I}+\mathcal{L}) \mathcal{P}_{1}$ is the desired left parametrix. In the latter steps we employed Theorem 6.9 several times.

7.3. Concluding remarks. Comparing the spaces $\mathcal{Y}^{\mu}\left(M,(\gamma, \gamma-\mu),\left(k, l, J_{-}, J_{+}\right)\right)_{S, T}$ and $\mathcal{Y}^{\mu}\left(M,(\gamma, \gamma-\mu),\left(k, l, J_{-}, J_{+}\right)\right)$, the additional ingredients of the new calculus are Green operators (in block-matrix sense) associated with discrete asymptotic data in the weight strips $\left\{z: \frac{n+1}{2}-\gamma<\right.$ $\left.\operatorname{Re} z<\frac{n+1}{2}-\gamma+\sigma\right\}$ and $\left\{z: \frac{n+1}{2}-(\gamma-\mu)<\operatorname{Re} z<\frac{n+1}{2}-(\gamma-\mu)+\tau\right\}$, respectively. Such operators are able to 'reproduce' non-smoothing Mellin operators in the upper left corner when they are given on the weight line $\operatorname{Re} z=\frac{n+1}{2}-\gamma+\sigma$ instead of $\operatorname{Re} z=\frac{n+1}{2}-\gamma$ (the latter is the case in both variants of the edge calculus). This is due to the fact that differences of Mellin operators for different weight lines (and with specific meromorphic symbols) generate Green operators of the singular category, cf. [3].

It would be interesting to study subalgebras of our calculus with meromorphic Mellin symbols in which the Mellin operators refer to $\operatorname{Re} z=\frac{n+1}{2}-\gamma+\sigma$ instead of $\operatorname{Re} z=\frac{n+1}{2}-\gamma$. This would require a careful choice of admitted Mellin symbols and a detailed analysis of the asymptotic data. The paper [8] of Liu and Witt can be interpreted in this spirit.

One may expect many other interesting examples of such subcalculi of edge problems, for instance, algebras generated by boundary value problems without the transmission property at the boundary, with (principal) interior symbols of the form $|\xi|^{\mu}$ for some $\mu \notin 2 \mathbb{Z}$ (the case $\mu \in 2 \mathbb{Z}$ corresponds to the case with transmission property). An edge algebra interpretation of boundary value problems (without transmission property) is given in [13].

Such investigations could deepen the insight into the structure of parametrices of mixed elliptic problems, but the details might be quite voluminuous.

\section{Appendix: The standard calculus for manifolds with edges}

In this appendix we shall give a brief summary of the so-called edge algebra. For a reference of the here presented material we refer the reader to $[\mathbf{3}],[\mathbf{5}]$, and $[\mathbf{1 5}]$.

In the following $\Omega \subset \mathbb{R}^{q}$ denotes an open set, and $X$ is a closed smooth manifold of dimension $n \in \mathbb{N}_{0}$. We shall denote by $L_{\mathrm{cl}}^{\mu}(X)$ the Fréchet space of classical pseudodifferential operators on $X$, and by $L_{\mathrm{cl}}^{\mu}(X ; \Lambda)$ the space of parameter-dependent operators, where $\Lambda=\mathbb{R}^{l}$ for some $l \in \mathbb{N}$. More precisely, the local symbols of parameter-dependent operators satisfy estimates of the form

$$
\left|\partial_{x}^{\beta} \partial_{\xi}^{\alpha} \partial_{\lambda}^{\gamma} a(x, \xi, \lambda)\right| \leq C(1+|\xi|+|\lambda|)^{\mu-|\alpha|-|\gamma|},
$$

and they have asymptotic expansions in components that are positively homogeneous in $(\xi, \lambda)$. In short, $(\xi, \lambda)$ is treated as a covariable.

8.1. Mellin symbols and Mellin pseudodifferential operators. For $\gamma \in \mathbb{R}$ and $\varepsilon>0$ let us set

$$
S_{(\gamma, \varepsilon)}=\left\{z \in \mathbb{C}:\left|\frac{1}{2}-\gamma-\operatorname{Re} z\right|<\varepsilon\right\}
$$


i.e. $S_{(\gamma, \varepsilon)}$ is the open, vertical strip of width $\varepsilon$ around the line $\operatorname{Re} z=\frac{1}{2}-\gamma$.

Definition 8.1. Let $\gamma \in \mathbb{R}, \mu \in \mathbb{R} \cup\{-\infty\}$ and $\varepsilon>0$. The space $M_{(\gamma, \varepsilon)}^{\mu}(X ; \Lambda)$ consists of all holomorphic functions $h: S_{\left(\gamma-\frac{n}{2}, \varepsilon\right)} \rightarrow L_{\mathrm{cl}}^{\mu}(X ; \Lambda)$ such that

$$
h_{\delta}(\lambda, \varrho):=h\left(\lambda, \frac{n+1}{2}-\gamma+\delta+i \varrho\right) \in L_{\mathrm{cl}}^{\mu}\left(X ; \Lambda \times \mathbb{R}_{\varrho}\right)
$$

uniformly in $\delta \in(-\varepsilon, \varepsilon)$. Since this is a Fréchet space in a canonical way, it is meaningful to speak of $C^{\infty}\left(\Omega \times \overline{\mathbb{R}}_{+}, M_{(\gamma, \varepsilon)}^{\mu}(X ; \Lambda)\right)$. We set

$$
M_{\gamma}^{\mu}(X ; \Lambda)=\cup_{\varepsilon>0} M_{(\gamma, \varepsilon)}^{\mu}(X ; \Lambda), \quad C^{\infty}\left(\Omega \times \overline{\mathbb{R}}_{+}, M_{\gamma}^{\mu}(X ; \Lambda)\right)=\cup_{\varepsilon>0} C^{\infty}\left(\Omega \times \overline{\mathbb{R}}_{+}, M_{(\gamma, \varepsilon)}^{\mu}(X ; \Lambda)\right),
$$

and

$$
M_{O}^{\mu}(X ; \Lambda)=\bigcap_{\varepsilon>0} M_{(\gamma, \varepsilon)}^{\mu}(X ; \Lambda), \quad C^{\infty}\left(\Omega \times \overline{\mathbb{R}}_{+}, M_{O}^{\mu}(X ; \Lambda)\right)=\bigcap_{\varepsilon>0} C^{\infty}\left(\Omega \times \overline{\mathbb{R}}_{+}, M_{(\gamma, \varepsilon)}^{\mu}(X ; \Lambda)\right) .
$$

The definitions are analogous for the spaces depending only on $\Omega \times \mathbb{R}_{+}$and those independent of the parameter $\lambda \in \Lambda$.

A symbol $h(y, r, \lambda, z)$ belonging to $C^{\infty}\left(\Omega \times \mathbb{R}_{+}, M_{\gamma}^{\mu}(X ; \Lambda)\right)$ induces a family of Mellin pseudodifferential operators on the infinite cone $X^{\wedge}=\mathbb{R}_{+} \times X$ by

$$
\left[\operatorname{op}_{M}^{\gamma-\frac{n}{2}}(h)(y, \lambda) u\right](r):=\frac{1}{2 \pi i} \int_{\operatorname{Re} z=\frac{n+1}{2}-\gamma} r^{-z} h(y, r, \lambda, z)(M u)(z) d z, \quad u \in C_{0}^{\infty}\left(X^{\wedge}\right) .
$$

Here, we have identified $C_{0}^{\infty}\left(X^{\wedge}\right)$ with $C_{0}^{\infty}\left(\mathbb{R}_{+}, C^{\infty}(X)\right)$, and $M$ denotes the Mellin transform.

8.2. Green and smoothing Mellin symbols. For the following definition recall the definition of cone Sobolev spaces $\mathcal{K}^{s, \gamma}\left(X^{\wedge}\right)$ from Section 2 and its group action $\kappa_{\lambda}$.

Definition 8.2. Let $\gamma, \gamma^{\prime} \in \mathbb{R}$ and $\mu \in \mathbb{R} \cup\{-\infty\}$. The space $R_{G}^{\mu}\left(\Omega \times \mathbb{R}^{q} ;\left(\gamma, \gamma^{\prime}\right)\right)$ consists of all operator-valued symbols (cf. Section 2) $g(y, \eta) \in S_{\mathrm{cl}}^{\mu}\left(\Omega \times \mathbb{R}^{q} ; \mathcal{K}^{0, \gamma}\left(X^{\wedge}\right), \mathcal{K}^{0, \gamma^{\prime}}\left(X^{\wedge}\right)\right)$ such that there exists an $\varepsilon>0$ with

$$
\begin{gathered}
g(y, \eta) \in \bigcap_{s \in \mathbb{R}} S_{\mathrm{cl}}^{\mu}\left(\Omega \times \mathbb{R}^{q} ; \mathcal{K}^{s, \gamma}\left(X^{\wedge}\right), \mathcal{S}^{\gamma^{\prime}+\varepsilon}\left(X^{\wedge}\right)\right), \\
g(y, \eta)^{*} \in \bigcap_{s \in \mathbb{R}} S_{\mathrm{cl}}^{\mu}\left(\Omega \times \mathbb{R}^{q} ; \mathcal{K}^{s,-\gamma^{\prime}}\left(X^{\wedge}\right), \mathcal{S}^{-\gamma+\varepsilon}\left(X^{\wedge}\right)\right) .
\end{gathered}
$$

In the previous definition, ${ }^{*}$ refers to the pointwise adjoint with respect to the scalar product of $\mathcal{K}^{0,0}\left(X^{\wedge}\right)=L_{2}\left(X^{\wedge}, r^{-n} d r d x\right)$, which allows an identification of $\mathcal{K}^{-s,-\gamma}\left(X^{\wedge}\right)$ and the dual space of $\mathcal{K}^{s, \gamma}\left(X^{\wedge}\right)$.

Definition 8.3. Let $\gamma \in \mathbb{R}$ and $\mu \in \mathbb{R} \cup\{-\infty\}$. The space $R_{M+G}^{\mu}\left(\Omega \times \mathbb{R}^{q} ;(\gamma, \gamma-\mu)\right)$ consists of all operator-valued symbols of the form

$$
m(y, \eta)+g(y, \eta)=\omega_{0}(r[\eta]) r^{-\mu} \mathrm{op}_{M}^{\gamma-\frac{n}{2}}(h)(y) \omega_{1}(r[\eta])+g(y, \eta),
$$

where $g(y, \eta) \in R_{G}^{\mu}\left(\Omega \times \mathbb{R}^{q} ;(\gamma, \gamma-\mu)\right)$ is a Green symbol, $h \in C^{\infty}\left(\Omega, M_{\gamma}^{-\infty}(X)\right)$, and $\omega_{0}, \omega_{1} \in$ $C^{\infty}([0,1[)$ are arbitrary cut-off functions. For $\nu \in \mathbb{R}$ with $1 \leq \mu-\nu \in \mathbb{N}$ we set

$$
R_{M+G}^{\nu}\left(\Omega \times \mathbb{R}^{q} ;(\gamma, \gamma-\mu)\right)=R_{G}^{\nu}\left(\Omega \times \mathbb{R}^{q} ;(\gamma, \gamma-\mu)\right) .
$$


It can be shown that then, for each $s \in \mathbb{R}$,

$$
R_{M+G}^{\nu}\left(\Omega \times \mathbb{R}^{q} ;(\gamma, \gamma-\mu)\right) \subset S_{\mathrm{cl}}^{\nu}\left(\Omega \times \mathbb{R}^{q} ; \mathcal{K}^{s, \gamma}\left(X^{\wedge}\right), \mathcal{K}^{\infty, \gamma-\mu}\left(X^{\wedge}\right)\right) .
$$

If $(m+g)(y, \eta)$ is as in (8.1), its homogeneous principal symbol is defined as

$$
\sigma_{\wedge}(m+g)(y, \eta)=\omega_{0}(r|\eta|) r^{-\mu} \operatorname{op}_{M}^{\gamma-\frac{n}{2}}(h)(y) \omega_{1}(r|\eta|)+\sigma_{\wedge}(g)(y, \eta), \quad \eta \neq 0,
$$

where $\sigma_{\wedge}(g)(y, \eta)$ denotes the homogeneous principal symbol of $g(y, \eta)$. This symbol we shall refer to as the principal edge symbol of $(m+g)(y, \eta)$. The (principal) conormal symbol of $(m+g)(y, \eta)$ is, by definition,

$$
\sigma_{M}(m+g)(y, z)=h(y, z) \in C^{\infty}\left(\Omega, M_{\gamma}^{-\infty}(X)\right)
$$

\subsection{Edge amplitude functions.}

Definition 8.4. Let $\gamma \in \mathbb{R}$ and $\mu, \nu \in \mathbb{R}$ with $\mu-\nu \in \mathbb{N}$. Then $R^{\nu}\left(\Omega \times \mathbb{R}^{q} ;(\gamma, \gamma-\mu)\right)$ denotes the space of so-called edge symbols

$$
a(y, \eta)=\sigma\left\{r^{-\nu} \operatorname{op}_{M}^{\gamma-n / 2}(h)(y, \eta)+m(y, \eta)+g(y, \eta)\right\} \sigma_{0}+(1-\sigma) \operatorname{op}(p)(y, \eta)\left(1-\sigma_{1}\right),
$$

where the notation has the following meaning:

a) $\sigma, \sigma_{0}, \sigma_{1} \in C_{0}^{\infty}\left(\left[0,1[)\right.\right.$ are cut-off functions with $\sigma_{1} \prec \sigma \prec \sigma_{0}$,

b) $h(y, r, \eta, z)=\widetilde{h}(y, r, r \eta, z)$ with $\widetilde{h} \in C^{\infty}\left(\Omega \times \overline{\mathbb{R}}_{+}, M_{O}^{\nu}\left(X ; \mathbb{R}_{\eta}^{q}\right)\right)$,

c) $(m+g)(y, \eta) \in R_{M+G}^{\nu}\left(\Omega \times \mathbb{R}^{q} ;(\gamma, \gamma-\mu)\right)$,

d) $p(y, r, \eta, \varrho) \in C^{\infty}\left(\Omega \times \mathbb{R}_{+}, L_{\mathrm{cl}}^{\nu}\left(X ; \mathbb{R}_{\eta}^{q} \times \mathbb{R}_{\varrho}\right)\right)$, independent of $r$ for large $r$.

Edge symbols are particular operator-valued symbols, namely

$$
R^{\nu}\left(\Omega \times \mathbb{R}^{q} ;(\gamma, \gamma-\mu)\right) \subset S^{\nu}\left(\Omega \times \mathbb{R}^{q} ; \mathcal{K}^{s, \gamma}\left(X^{\wedge}\right), \mathcal{K}^{s-\nu, \gamma-\mu}\left(X^{\wedge}\right)\right) .
$$

In case $\nu=\mu$ we define the principal edge symbol of $a(y, \eta)$ by

$$
\sigma_{\wedge}(a)(y, \eta)=r^{-\mu} \mathrm{op}_{M}^{\gamma-n / 2}\left(h_{\wedge}\right)(y, \eta)+\sigma_{\wedge}(m+g)(y, \eta), \quad h_{\wedge}(y, r, z, \eta)=\widetilde{h}(y, 0, r \eta, z),
$$

cf. (8.2), and the conormal symbol of $a(y, \eta)$ by

$$
\sigma_{M}(a)(y, z)=\widetilde{h}(y, 0, z, 0)+\sigma_{M}(m+g)(y, z),
$$

cf. (8.3).

There is an obvious generalization to $(l \times k)$-matrices of such edge symbols, yielding the space

$$
R^{\nu}\left(\Omega \times \mathbb{R}^{q} ;(\gamma, \gamma-\mu),(k, l)\right) .
$$

8.4. The algebra of edge pseudodifferential operators on $M$. In the following we let $M$ be a manifold with edge $Y$ and model cone $X^{\wedge}$ as it is described in Section 6 . For $\gamma \in \mathbb{R}$ and $\mu, \nu \in \mathbb{R}$ with $\mu-\nu \in \mathbb{N}$,

$$
Y^{\nu}(M,(\gamma, \gamma-\mu),(k, l))
$$

denotes the space of $\nu$-th order edge operators (with respect to $(k, l)$ and the weight-data $(\gamma, \gamma-\mu)$ ). Modulo global smoothing operators they are obtained by pasting together (using a partition of unity on $M$ ) usual pseudodifferential operators of order $\nu$ on the interior of $M$ with operators that are localized near the edge and that are defined locally by means of pseudodifferential operators with operator-valued symbols from $R^{\nu}\left(\Omega \times \mathbb{R}^{q} ;(\gamma, \gamma-\mu),(k, l)\right)$. We shall omit the details here. 
The space of global smoothing operators, $Y^{-\infty}(M,(\gamma, \gamma-\mu),(k, l))$, consists of all operators $G$ : $\mathcal{W}^{0, \gamma}\left(M, \mathbb{C}^{k}\right) \rightarrow \mathcal{W}^{0, \gamma-\mu}\left(M, \mathbb{C}^{l}\right)$ such that there exists an $\varepsilon>0$ with

$$
G: \mathcal{W}^{s, \gamma}\left(M, \mathbb{C}^{k}\right) \longrightarrow \mathcal{W}^{\infty, \gamma-\mu+\varepsilon}\left(M, \mathbb{C}^{l}\right), \quad G^{*}: \mathcal{W}^{s,-\gamma+\mu}\left(M, \mathbb{C}^{l}\right) \longrightarrow \mathcal{W}^{\infty,-\gamma+\varepsilon}\left(M, \mathbb{C}^{k}\right)
$$

for each $s \in \mathbb{R}$, where ${ }^{*}$ refers to the adjoint with respect to the scalar product of $\mathcal{W}^{0,0}(M)$ that admits an identification of the dual space of $\mathcal{W}^{s, \sigma}(M)$ with $\mathcal{W}^{-s,-\sigma}(M)$.

Each $A \in Y^{\nu}(M,(\gamma, \gamma-\mu),(k, l))$ induces continuous operators

$$
\mathcal{W}^{s, \gamma}\left(M, \mathbb{C}^{k}\right) \longrightarrow \mathcal{W}^{s-\nu, \gamma-\mu}\left(M, \mathbb{C}^{l}\right), \quad s \in \mathbb{R} .
$$

8.5. Principal symbols. As a matter of fact,

$$
Y^{\nu}(M,(\gamma, \gamma-\mu),(k, l)) \subset L_{\mathrm{cl}}^{\nu}\left(\operatorname{int} M ; \mathbb{C}^{k}, \mathbb{C}^{l}\right)
$$

for int $M:=M \backslash Y$, i.e., any edge operator is a usual pseudodifferential operator on the interior of $M$. In particular, with each $A \in Y^{\mu}(M,(\gamma, \gamma-\mu),(k, l))$ we can associate the standard homogeneous principal symbol

$$
\sigma_{\psi}(A) \in C^{\infty}\left(T^{*} \operatorname{int} M \backslash 0, \mathcal{L}\left(\mathbb{C}^{k}, \mathbb{C}^{l}\right)\right) .
$$

In local coordinates, corresponding to the splitting of coordinates $(y, r, x)$ near the edge, the homogeneous principal symbol has a special 'degenerate' form namely

$$
\sigma_{\psi}(A)(y, r, x, \eta, \varrho, \xi)=r^{-\mu} \widetilde{p}_{(\mu)}(y, r, x, r \eta, r \varrho, \xi),
$$

with a symbol $\widetilde{p}_{(\mu)}(y, r, x, \widetilde{\eta}, \widetilde{\varrho}, \xi)$ homogeneous in $(\widetilde{\eta}, \widetilde{\varrho}, \xi)$. Removing this degeneracy leads to

$$
\widetilde{\sigma}_{\psi}(A)(y, x, \eta, \varrho, \xi)=\widetilde{p}_{(\mu)}(y, 0, x, \eta, \varrho, \xi)=\lim _{r \rightarrow 0+} r^{\mu} \sigma_{\psi}(A)\left(y, r, x, r^{-1} \eta, r^{-1} \varrho, \xi\right) .
$$

Globally, this yields the so-called rescaled symbol

$$
\widetilde{\sigma}_{\psi}(A) \in C^{\infty}\left(\left(T^{*}(Y \times X) \times \mathbb{R}\right) \backslash 0, \mathcal{L}\left(\mathbb{C}^{k}, \mathbb{C}^{l}\right)\right) .
$$

Moreover, induced by the local amplitude functions of Definition 8.4, we have the principal edge symbol

$$
\sigma_{\wedge}(A) \in C^{\infty}\left(T^{*} Y \backslash 0, \mathcal{L}\left(\mathcal{K}^{s, \gamma}\left(X^{\wedge}, \mathbb{C}^{k}\right), \mathcal{K}^{s-\mu, \gamma-\mu}\left(X^{\wedge}, \mathbb{C}^{l}\right)\right)\right.
$$

8.6. Ellipticity. An operator $\mathcal{A} \in \mathcal{Y}^{\mu}\left(M,(\gamma, \gamma-\mu),\left(k, k ; J_{-}, J_{+}\right)\right)$(cf. the notation of Remark $6.7)$ is said to be elliptic, if $\sigma_{\psi}(\mathcal{A}), \widetilde{\sigma}_{\psi}(\mathcal{A})$, and $\sigma_{\wedge}(A)$ satisfy the conditions of Definition 7.1.

TheOREM 8.5. An elliptic operator $\mathcal{A} \in \mathcal{Y}^{\mu}\left(M,(\gamma, \gamma-\mu),\left(k, k ; J_{-}, J_{+}\right)\right)$possesses a parametrix $\mathcal{P} \in \mathcal{Y}^{-\mu}\left(M,(\gamma-\mu, \gamma),\left(k, k ; J_{+}, J_{-}\right)\right)$, i.e. $\mathcal{A} \mathcal{P}-\mathcal{I}$ and $\mathcal{P} \mathcal{A}-\mathcal{I}$ are of order $-\infty$ in the spaces belonging to the data $\left((\gamma-\mu, \gamma-\mu),\left(k, k ; J_{+}, J_{+}\right)\right)$and $\left((\gamma, \gamma),\left(k, k ; J_{-}, J_{-}\right)\right)$, respectively.

For a proof of this theorem see [3], for example. 


\section{References}

[1] L. Boutet de Monvel. Boundary problems for pseudo-differential operators. Acta Math. 126 (1971), 11-51.

[2] N. Dines, B.-W. Schulze. Mellin-edge-representations of elliptic operators. Math. Methods Appl. Sci. 28 (2005), $2133-2172$.

[3] Ju.V. Egorov, B.-W. Schulze. Pseudo-differential operators, singularities, applications. Operator Theory: Advances and Applications 93, Birkhäuser Verlag, 1997.

[4] G.I. Eskin. Boundary value problems for elliptic pseudodifferential equations. Math. Monographs 52, Amer. Math. Soc., 1980.

[5] J.B. Gil, B.-W. Schulze, and J. Seiler. Cone pseudodifferential operators in the edge symbolic calculus. Osaka J. Math. 37 (2000), 221-260.

[6] G. Harutjunjan, B.-W. Schulze. Elliptic Mixed, Transmission, and Singular Crack Problems. European Math. Soc. Publ. To appear 2006.

[7] D. Kapanadze, B.-W. Schulze. Crack theory and edge singularities. Mathematics and its Applications 561, Kluwer Academic Publishing, 2003.

[8] X. Liu, I. Witt. Pseudodifferential calculi on the half-line respecting prescribed asymptotic types. Integral Equations Opeartor Theory 49 (2004), 473-497.

[9] M.I. Vishik, G.I. Eskin, Convolution equations in a bounded region, Uspekhi Mat. Nauk 20 (1965), 89-152.

[10] S. Rempel, B.-W. Schulze. Parametrices and boundary symbolic calculus for elliptic boundary problems without the transmission property. Math. Nachr. 105 (1982), 45-149.

[11] B.-W. Schulze. Pseudo-differential operators on manifolds with singularities. North-Holland, 1991.

[12] B.-W. Schulze. Pseudo-differential operators on manifolds with edges. In Symp. "Partial Differential Equations", Holzhau 1988. Teubner-Texte Math. 112, pp. 259-287. Teubner Verlag, 1989.

[13] B.-W. Schulze, J. Seiler. The edge algebra structure of boundary value problems. Ann. Global Anal. Geom. 22 (2002), 197-265.

[14] B.-W. Schulze, J. Seiler. Pseudodifferential boundary value problems with global projection conditions. J. Funct. Anal. 206 (2004), 449-498.

[15] J. Seiler. Pseudodifferential calculus on manifolds with non-compact edges. Dissertation, University of Potsdam, 1997.

[16] N. Tarkhanov. Harmonic integrals on domains with edges. Preprint 2002/02, University of Potsdam.

[17] I. Witt. Explicit algebras with the Leibniz-Mellin translation product. Preprint 1999/02, University of Potsdam.

A. Razmadze Mathematical Institute, Academy of Sciences of Georgia, M. Alexidze Str. 1, Tbilisi 0193, GEORGIA

E-mail address: daka@rmi.acnet.ge

Universität Potsdam, Institut für Mathematik, Postfach 601553, 14415 Potsdam, Germany

E-mail address: schulze@math.uni-potsdam.de

Universität Hannover, Institut für Angewandte Mathematik, Welfengarten 1, 30167 Hannover, GerMANY

E-mail address: seiler@ifam.uni-hannover.de 